\title{
A novel differential evolution algorithm approach for estimating the parameters of Gamma distribution: An application to the failure stresses of single carbon fibres
}

\author{
Aynur Yonar (D), Nimet Yapıcı Pehlivan (D)* \\ Department of Statistics, Faculty of Science, Selcuk University, Konya, Turkey
}

\begin{abstract}
Three-parameter (3-p) Gamma distribution is widely used to model for skewed data in the reliability field. Thus, the problem of parameter estimation for the Gamma distribution has remained significant and interesting in all times. The maximum likelihood (ML) and the least square (LS) are the most popular methods in the parameter estimation. In this study, a novel Differential Evolution (DE) algorithm is proposed for the ML and LS estimation of the parameters of the 3-p Gamma distribution. This approach overcomes the problem of how to determine the search space of the DE by utilizing a new search space based on the confidence interval. The modified maximum likelihood and the profile likelihood methods are considered to constitute the confidence interval. In order to examine the performance of the proposed approach, an extensive Monte Carlo simulation study and a real data application are performed. The results show that this proposed approach is effective for estimating the parameters of the 3-p Gamma distribution with respect to mean square error and deficiency criteria.
\end{abstract}

Mathematics Subject Classification (2010). 62F07,62F10,62P30

Keywords. Differential evolution, Gamma distribution, Maximum likelihood, Least square, Monte carlo simulation, Parameter estimation

\section{Introduction}

Three parameters (3-p) Gamma distribution is one of the commonly used distributions for modeling skewed data in the field of reliability, hydrology, and finance $[3,6,12,18]$. The probability density function and cumulative distribution function of the 3-p Gamma distribution are defined by

$$
f(x ; \sigma, \beta, \mu)=\frac{1}{\Gamma(\sigma) \beta^{\sigma}}(x-\mu)^{\sigma-1} \exp \left(-\frac{x-\mu}{\beta}\right), \quad x \geq \mu, \sigma>0, \beta>0 .
$$

\footnotetext{
*Corresponding Author.

Email addresses: aynursahin@selcuk.edu.tr (A. Yonar), nimet@selcuk.edu.tr (N. Yapıcı Pehlivan)

Received: 14.02.2020; Accepted: 14.06.2020
} 
and

$$
F(x ; \sigma, \beta, \mu)=\frac{1}{\Gamma(\sigma)} \gamma\left(\sigma, \frac{x-\mu}{\beta}\right), \quad x \geq \mu, \sigma>0, \beta>0
$$

respectively. In Equations (1.1) and (1.2), $\sigma$ is the shape parameter, $\beta$ is the scale parameter, $\mu$ is the location parameter, and $\gamma$ indicates the incomplete Gamma function $[3$, 11].

3-p Gamma distribution contains three special cases according to the values of the shape parameter $\sigma$. 3-p Gamma distribution is " $J$ " shaped for $\sigma \leq 1$ and bell-shaped for $\sigma>1$. The distribution becomes an exponential distribution if $\sigma=1$. Therefore, it is one of the common distributions used in the statistical modeling $[4,18]$.

The successful applications of the 3-p Gamma distribution depend on having acceptable statistical estimates of its parameters. However, parameter estimation for the 3-p Gamma distribution is a quite difficult problem. There are various studies for the parameter estimation of the 3-p Gamma distribution in the literature. Cohen and Whitten [6] proposed a modification of the maximum likelihood (ML) and moment estimators for parameters of the 3-p Gamma distribution and they revealed that the new estimators are better than both the ML and the moment estimators in terms of bias, variance, and ease of calculation. Cohen and Whitten [7] presented modified moment estimators by replacing the third moment with a function of the first-order statistic and they showed that these estimators are computationally simpler than ML even when samples are small. Hirose [10] suggested a new ML parameter estimation scheme by utilizing the reparametrized distribution function and predictor-corrected method and the effectiveness of the proposed method was showed by a Monte Carlo simulation study. Balakrishnan and Wang [3] presented some simple efficient estimators by using the first few order statistic and they indicated that these estimators are more efficient than Cohen and Whitten [7]'s method. Tzavelas [27] introduced a program in Mathematica to obtain the solutions of the likelihood equations in the ML parameter estimation for the 3-p Gamma distributions. Lakshmi and Vaidyanathan [12] proposed a novel approach for the ML, the least square (LS) and the maximum product spacing (MPS) methods to estimate the parameters of the 3-p Gamma distribution by using an extensive search over three-dimensional parameter space.

The ML and the LS are the two most commonly used methods for parameter estimation. Due to the fact that these methods don't provide explicit estimators for the parameters of 3-p Gamma distributions, iterative methods are needed [1].

In this study, differential evolution (DE) algorithm, which is one of the most powerful evolutionary algorithms (EAs) for the continuous parameter spaces in recent times, is used to obtain the ML and LS estimators of the parameters of 3-p Gamma distribution. To the best of our knowledge, this is the first study to use the DE for estimating the parameters of the 3-p Gamma distribution. In addition, there is no study using another metaheuristic method for this in the literature.

The performance of the DE is highly affected by initial search space. Arbitrary determination of the initial search space can cause some following problems. (i) if the search space is determined too wide, the algorithm may provide unacceptable results, or (ii) if the search space is determined too narrow, this search space may not contain the actual values which are sought. To overcome these problems, we propose a new approach to construct search space for the parameters of the 3-p Gamma distribution by utilizing confidence intervals based on the modified maximum likelihood (MML) estimators and the profile likelihood (PL) method. The MML method was proposed by Tiku and Akkaya [26] and MML estimators can be written in explicit form as a function of observations for 
the parameters of $\beta$ and $\mu$ when $\sigma$ is known. In this case, the PL method can be used for estimating the shape parameter $\sigma$. Thus, confidence intervals can be obtained through the MML and the PL methods.

The rest of this paper is organized as follows: In Section 2, the ML and the LS estimations process for the parameters of the 3-p Gamma distribution are introduced and the proposed DE approach is presented. In Section 3, an extensive Monte Carlo simulation study is conducted, and the results of the simulation are given. In Section 4, a real-life application is examined. Conclusions are presented in the final section.

\section{Parameter estimation for the parameters of Gamma distribution by DE algorithm}

In this section, the ML and the LS estimators of parameters of the 3-p Gamma distribution by the DE algorithm are discussed. After the DE algorithm is introduced briefly, the notions of the ML and LS estimators of the 3-p Gamma distribution parameters are given and the proposed approach is presented by explaining the Modified Maximum Likelihood (MML) and Profile Likelihood (PL) methods.

\subsection{The DE algorithm}

The Differential Evolution (DE), which is proposed by Storn [23] and Storn and Price [24], is one of the most powerful evolutionary algorithms which are mainly inspired from the biological process of evolution [2]. Due to its easy implementation, strong global search ability and fast convergence speed, DE has become one of the most popular optimization techniques and it has been widely used for solving the nonlinear and complex problems in recent years $[9,13,15-17,22,32]$.

Although the DE algorithm has only three control parameters which are scaling factor, crossover factor and population size, it performs remarkably in terms of accuracy, computation speed and robustness while optimizing various objective functions [9].

The DE algorithm consists of three basic steps: mutation, crossover and selection. After initialization, the DE enters an evolutionary process including mutation, crossover, and selection operations as described as following $[19,21]$ :

\section{Initialization}

For a D-dimensional optimization problem, the DE algorithm searches the global optimum point by using the vector $\vec{x}_{i}^{g}=\left[x_{1, i}^{g}, x_{2, i}^{g}, \ldots, x_{D, i}^{g}\right]$ which is represented the vector $i$ of the population at current generation $g$. Each vector, also known as genome/chromosome, forms a candidate solution to the multidimensional optimization problem.

The initial population is generated by using the prescribed minimum and maximum parameter bounds $\left(x_{j, \min }\right)$ and $\left(x_{j, \max }\right)$, which are known as search space. The initial value of the $j$ th component of the vector $i$ at $g=0$ is generated by following:

$$
x_{j, i}^{0}=x_{j, \min }+\operatorname{rand}_{i j}[0,1]\left\{x_{j, \max }-x_{j, \min }\right\}
$$

In Equation $(2.1), \operatorname{rand}_{i j}[0,1]$ represents a uniformly distributed random variable in the range $[0,1][25,31]$.

\section{Mutation}

The DE applies the mutation operator to create a donor vector $\left(\vec{v}_{i}^{g}\right)$, after initialization. There are different mutation strategies in the literature. The general notation used for naming the various mutation strategies is $\mathrm{DE} / \mathrm{x} / \mathrm{y} / \mathrm{z}$, where $\mathrm{DE}$ stands for differential 
evolution, $\mathrm{x}$ specifies the vector to be mutated, and $\mathrm{y}$ is the number of difference vectors, $z$ denotes the type of crossover used (exp: exponential and bin: binomial) [25, 31]. In this study, the standard variant of the DE, i.e. DE/rand/1/bin, has been used.

For each vector of the population, three distinct vectors $r_{1}, r_{2}, r_{3} ; r_{1} \neq r_{2} \neq r_{3} \neq i \in$ $(0, N P)$ is randomly chosen and a new donor vector $\left(\vec{v}_{i}^{g}\right)$ by mutation scheme is generated as follows:

$$
\vec{v}_{i}^{g}=\vec{x}_{r 1}^{g}+F\left(\vec{x}_{r 2}^{g}-\vec{x}_{r 3}^{g}\right)
$$

In Equation (2.2), $F$ is the scaling factor which is used to scale the difference vectors.

\section{Crossover}

Crossover operation is applied to each pair of the target vector $\vec{x}_{i}^{g}$ and its corresponding mutant vector $\vec{v}_{i}^{g}$ to generate a trial vector, after generating the new donor vector. A random index $j_{\text {rand }} \in[0, D]$ is generated and the crossover operator is applied for increasing the diversity of the population by following:

$$
\vec{u}_{j, i}^{g+1}= \begin{cases}\vec{v}_{j, i}^{g}, & \text { if } \text { rand }_{i, j}[0,1] \leq C R \text { or } j=j_{\text {rand }} \\ \vec{x}_{j, i}^{g}, \text { otherwise }\end{cases}
$$

In Equation (2.3), $\operatorname{rand}_{i, j}[0,1]$ represents a uniformly distributed random variable in the range $[0,1] . C R \in[0,1]$ is a crossover rate which is a user-defined constant, $j_{\text {rand }} \in[1,2, \ldots, D]$ is randomly chosen index used to ensure that at least one of the components comes from $\vec{v}_{i}^{g}$.

\section{Selection}

Selection operator is used to determine target vector $\left(\vec{x}_{i}^{g}\right)$ and trial vector $\left(\vec{u}_{i}^{g}\right)$ which will be transferred to the next generation. The selection scheme given by Equation (2.4) is applied for determining the solutions to be transferred to the next generation.

$$
\vec{x}_{i}^{g+1}= \begin{cases}\vec{u}_{i}^{g}, & f\left(\vec{u}_{i}^{g}\right) \geq f\left(\vec{x}_{i}^{g}\right) \\ \vec{x}_{i}^{g}, & f\left(\vec{u}_{i}^{g}\right)<f\left(\vec{x}_{i}^{g}\right)\end{cases}
$$

In Equation (2.4), $f$ is the objective function to be maximized. The vector transferred to the next generation is better than the competing vector, and such a selection guarantees that the global best of the population is transferred to the next generation all the time. In other words, the elitist strategy is implemented as the DE selection strategy.

\subsection{Maximum likelihood estimation}

Let $x_{1}, x_{2}, \ldots, x_{n}$ be a random variable sample drawn from the Gamma distribution expressed as $\operatorname{Gamma}(\sigma, \beta, \mu)$. The $\log$-likelihood function, $\log L(\theta / \underline{x})$ of the gamma distribution is given by

$$
\log L(\theta / \underline{x})=(\sigma-1) \sum_{i=1}^{n} \log \left(x_{i}-\mu\right)-\sum_{i=1}^{n}\left(\frac{x_{i}-\mu}{\beta}\right)-n \log \Gamma(\sigma)-n \sigma \log \beta
$$


where $\theta=(\sigma, \beta, \mu)$ is a vector of representing unknown parameters [4, 12]. To obtain the ML estimates of the parameters $\sigma, \beta$ and $\mu$, the likelihood function (or the log likelihood function) is maximized as follows:

$$
\begin{gathered}
\max _{\theta=(\sigma, \beta, \mu)} \log L(\theta=(\sigma, \beta, \mu) / \underline{x}) \\
\mu \leq \min (\underline{x}) \\
\sigma \geq 0, \beta \geq 0
\end{gathered}
$$

\subsection{Least square estimation}

Let $x_{(1)} \leq x_{(2)} \leq \ldots \leq x_{(n)}$ be the observed values of the order statistics taken from population which have $\operatorname{Gamma}(\sigma, \beta, \mu)$ distribution.

$$
F\left(x_{(i)}\right)=\frac{1}{\Gamma(\sigma)} \gamma\left(\sigma, \frac{x_{(i)}-\mu}{\beta}\right), \quad x_{(i)} \geq \mu, \sigma>0, \beta>0
$$

If the empirical distribution function is written instead of $F\left(x_{(i)}\right)$ in Equation (2.7), the model can be written as follows:

$$
\frac{i}{n+1}=F\left(x_{(i)}\right)+\varepsilon_{i}, i=1,2, \ldots, n
$$

Sum of squares of error terms $\varepsilon_{i}$ is minimized to obtain the LS estimates of the parameters $\sigma, \beta$ and $\mu$ as follows:

$$
\begin{gathered}
\min _{\theta=(\sigma, \beta, \mu)} \sum_{i=1}^{n} \varepsilon_{i}^{2}=\min _{\theta=(\sigma, \beta, \mu)} \sum_{i=1}^{n}\left(\frac{i}{n+1}-F\left(x_{(i)}\right)\right)^{2} \\
\mu \leq \min (\underline{x}) \\
\sigma \geq 0, \beta \geq 0
\end{gathered}
$$

Such problems given in Equation (2.9) can be solved by taking the first partial derivatives of the objective function according to unknown parameters and equalizing to zero. Because the equations that contain nonlinear function cannot be solved, it is recommended that using metaheuristic algorithms to solve the optimization problems in Equation (2.6) and (2.9). Therefore, a novel DE algorithm is proposed to find the ML and LS estimates of the unknown parameters of the 3-p Gamma distribution in this study. The DE is chosen in this study because it is one of the well-known and the most effective evolutionary algorithms for optimization problems and it has obvious advantages including simplicity to implementation, robustness, reliability [13, 14, 19]. Furthermore, Özsoy et al. [20] showed DE gives better performance than other metaheuristic methods such as PSO, SA, GA in terms of bias, variance, MSE values of the parameter estimations. 


\subsection{A novel DE algorithm approach based on modified maximum likeli- hood and Profile Likelihood}

The DE algorithm is considered for solving the optimization problems to find the ML and the LS estimates of the parameters of the 3-p Gamma distribution in this study. However, the performance of this algorithm is highly influenced by search space similar to other meta-heuristic methods. Previously, Örkcü, Aksoy [19] used the DE algorithm by arbitrarily determining the search space for estimating the parameters of the 3-p Weibull distribution.

In this study, we proposed a novel method to construct confidence intervals for each parameter which will be used as search space of the DE algorithm, by using Modified Maximum Likelihood (MML) and Profile Likelihood (PL) methods. The MML method is handled since the MML estimators for the 3-p Gamma distribution can be obtained in a clear form, namely written as a function of the observations.

The methodologies of the MML and the PL are given below.

\section{Modified maximum likelihood for the 3-p Gamma distribution}

To obtain the MML estimates of the parameters for the 3-p Gamma distribution, the following steps are used:

Step 1: The standardized observations $z_{i}=\left(x_{i}-\mu\right) / \beta$ are sorted in ascending order of magnitude as $z_{(1)} \leq z_{(2)} \leq \cdots \leq z_{(n)}$.

Step 2: The likelihood equations are written using the rank statistics.

$$
\begin{aligned}
& \frac{\partial \log L}{\partial \sigma}=\sum_{i=1}^{n} \log \left(z_{(i)}\right)-\frac{n}{\beta} \psi(\sigma)-\frac{n}{\beta} \log \beta=0, \\
& \frac{\partial \log L}{\partial \beta}=\frac{1}{\beta} \sum_{i=1}^{n} z_{(i)}-\frac{\sigma-1}{\beta} \sum_{i=1}^{n} z_{(i)} w\left(z_{(i)}\right)-\frac{n}{\beta}=0 . \\
& \frac{\partial \log L}{\partial \mu}=-\frac{(\sigma-1)}{\beta} \sum_{i=1}^{n} w\left(z_{(i)}\right)+\frac{n}{\beta}=0 .
\end{aligned}
$$

In Equation (2.10), $w(z)=z^{-1}, z_{(i)}=\left(x_{(i)}-\mu\right) / \beta$ and $\sum_{i=1}^{n} z_{(i)}=\sum_{i=1}^{n} z_{i}$.

Step 3: Let $t_{(i)}=E\left(z_{(i)}\right)$ be the expected value of ordered standardized variable $z_{(i)}$. A linear approximation of the nonlinear function $w\left(z_{(i)}\right)$ using the first two terms of Taylor series expansion around $t_{(i)}$ is as follows:

$$
\begin{aligned}
w\left(z_{(i)}\right) & \cong w\left(t_{(i)}\right)+\left(z_{(i)}-t_{(i)}\right)\left\{\frac{\partial}{\partial z} w(z)\right\}_{z=t_{(i)}} \\
= & \lambda_{i}-\gamma_{i} z_{(i)}, \quad i=1,2, \ldots, n
\end{aligned}
$$

In Equation (2.11);

$$
\begin{gathered}
\gamma_{i}=-\left.\frac{\partial}{\partial z} w(z)\right|_{z=t_{(i)}}=-\left.\frac{\partial}{\partial z} z^{-1}\right|_{z=t_{(i)}}=\frac{1}{t_{(i)}^{2}}, \\
\lambda_{i}=w\left(t_{(i)}\right)+t_{(i)} \gamma_{i}=\frac{1}{t_{(i)}}+t_{(i)} \frac{1}{t_{(i)}^{2}}=\frac{2}{t_{(i)}} .
\end{gathered}
$$


Despite the fact that the exact values of $t_{(i)}$ are not available, approximate values can be obtained from the solution of equation $\int_{-\propto}^{t_{(i)}} f(z) d z=\frac{i}{n+1}, 1 \leq i \leq n . f($.$) indicates$ the probability density function of the 3-p Gamma distribution.

Step 4: The modified likelihood equations are obtained by inserting Equation (2.11) into the Equation (2.10) as follows:

$$
\begin{aligned}
& \frac{\partial \log L}{\partial \sigma}=\sum_{i=1}^{n} \log \left(z_{(i)}\right)-\frac{n}{\beta} \psi(\sigma)-\frac{n}{\beta} \log \beta=0, \\
& \frac{\partial \log L}{\partial \beta}=\frac{1}{\beta} \sum_{i=1}^{n} z_{(i)}-\frac{\sigma-1}{\beta} \sum_{i=1}^{n} z_{(i)}\left(\lambda_{i}-\gamma_{i} z_{(i)}\right)-\frac{n}{\beta}=0 . \\
& \frac{\partial \log L}{\partial \mu}=-\frac{(\sigma-1)}{\beta} \sum_{i=1}^{n}\left(\lambda_{i}-\gamma_{i} z_{(i)}\right)+\frac{n}{\beta}=0 .
\end{aligned}
$$

Step 5: The MML estimators are obtained by solving the modified likelihood equations with regard to the parameters of $\mu$ and $\beta$, as follows, respectively:

$$
\begin{gathered}
\hat{\mu}_{M M L}=K+D \hat{\sigma}_{M M L} \\
\hat{\beta}_{M M L}=\frac{-B+\sqrt{B^{2}+4 n C}}{2 n}
\end{gathered}
$$

In Equations (2.15) and (2.16), $K=\frac{1}{m} \sum_{i=1}^{n} \gamma_{i} y_{[i]}, m=\sum_{i=1}^{n} \gamma_{i}, D=\frac{1}{m} \sum_{i=1}^{n} \lambda_{i}$, $B=\sum_{i=1}^{n} \lambda_{i}\left(y_{i}-K\right)$, and $C=\sum_{i=1}^{n} \gamma_{i}\left(y_{(i)}-K\right)^{2}=\sum_{i=1}^{n} \gamma_{i} y_{(i)}{ }^{2}-m K^{2}$.

Because the MML estimators given in Equations (2.15) and (2.16) are the functions of the sample observations, it is easy to compute the MML estimators. Furthermore, the MML estimators are robust, unbiased and minimum variance bound estimators under certain mild regularity conditions [29].

As the MML estimators asymptotically equal to the ML estimators, the asymptotic variance covariance matrix $I^{-1}(\mu, \beta)$ is equal to inverse of the Fisher information matrix $I(\mu, \beta)$. Thus, asymptotic variances of $\hat{\mu}_{M M L}$ and $\hat{\beta}_{M M L}$ for $\sigma>2$ are given as follows, respectively [26]

$$
\begin{gathered}
V\left(\hat{\mu}_{M M L}\right)=\frac{\sigma(\sigma-2) \beta^{2}}{2 n} \\
V\left(\hat{\beta}_{M M L}\right)=\frac{\beta^{2}}{2(n-3)}
\end{gathered}
$$

Confidence intervals with asymptotic $(1-\alpha)$ significance level for the position and the scale parameters are defined by following, respectively 


$$
\begin{aligned}
& \hat{\mu}_{M M L}-z_{\alpha / 2} \sqrt{V\left(\hat{\mu}_{M M L}\right)}<\mu<\hat{\mu}_{M M L}-z_{\alpha / 2} \sqrt{V\left(\hat{\mu}_{M M L}\right)} \\
& \hat{\beta}_{M M L}-z_{\alpha / 2} \sqrt{V\left(\hat{\beta}_{M M L}\right)}<\beta<\hat{\beta}_{M M L}-z_{\alpha / 2} \sqrt{V\left(\hat{\beta}_{M M L}\right)}
\end{aligned}
$$

The MML estimators have three desirable properties which are best asymptotically normality, almost fully efficiency for small samples, and explicit functions of sample observations for easy computation $[5,26]$.

The MML estimators can be obtained under the assumption that the shape parameter $\sigma$ is known. Therefore, the Profile Likelihood (PL) method is used to estimate the shape parameter $\sigma[28,30]$.

\section{Profile likelihood method for the 3-p Gamma distribution}

The steps of the PPL method for this study are given below.

Step 1: Calculate the $\hat{\mu}_{M M L}$ and $\hat{\beta}_{M M L}$ for a given value of $\sigma$ by using the Equation (2.15) and (2.16), respectively.

Step 2: Calculate the log-likelihood function value $\log L\left(\theta=\left(\sigma, \hat{\mu}_{M M L}, \hat{\beta}_{M M L}\right) / \underline{x}\right)$ given Equation (2.5).

Step 3: Repeat the Step 1 and Step 2 for different $\sigma$ values.

Step 4: The value of $\sigma$ that maximizes the log likelihood function is determined as the possible estimate for the shape parameter $\sigma$.

Confidence interval with asymptotic $(1-\alpha)$ significance level for shape parameter $\sigma$ is constituted as follows:

$$
\hat{\sigma}-z_{\alpha / 2} \sqrt{V(\hat{\sigma})}<\sigma<\hat{\sigma}+z_{\alpha / 2} \sqrt{V(\hat{\sigma})}
$$

The search space of the location, scale and shape parameters of the 3-p Gamma distribution are constructed by Equations (2.19), (2.20) and (2.21), respectively.

The flowchart for the proposed DE approach is illustrated in Figure 1.

\section{Simulation study}

In this section, Monte-Carlo simulation study is conducted to investigate the performance of the proposed approach for the methods of ML and the LS. Actual parameter values for the 3-p Gamma distribution are thought to be $\theta=(3,1,-2), \theta=(4,0.5,10)$ and $\theta=(2.56,0.75,8)$. In addition, sample sizes $10,30,50,100,250,500$, and 1000 are considered for each parameter in order to investigate the effect of sample size on parameter estimation process.

A scaling factor $F \in(0,1]$ and a crossover factor $C_{r}=2$ are considered for the DE algoithm. Population sizes (pop) are considered as 10, 25 and, 50. In the proposed approach, search space is obtained using confidence intervals via the MML and PL methods. In addition, the effectiveness of estimates for different search spaces are compared by using the arbitrarily specified search spaces shown in Table 3.1.

Parameter estimations are obtained by simulations. Each simulation structure is repeated 1000 times, and $3 \times 7 \times 3 \times 1000=63.000$ independent experiments are performed 


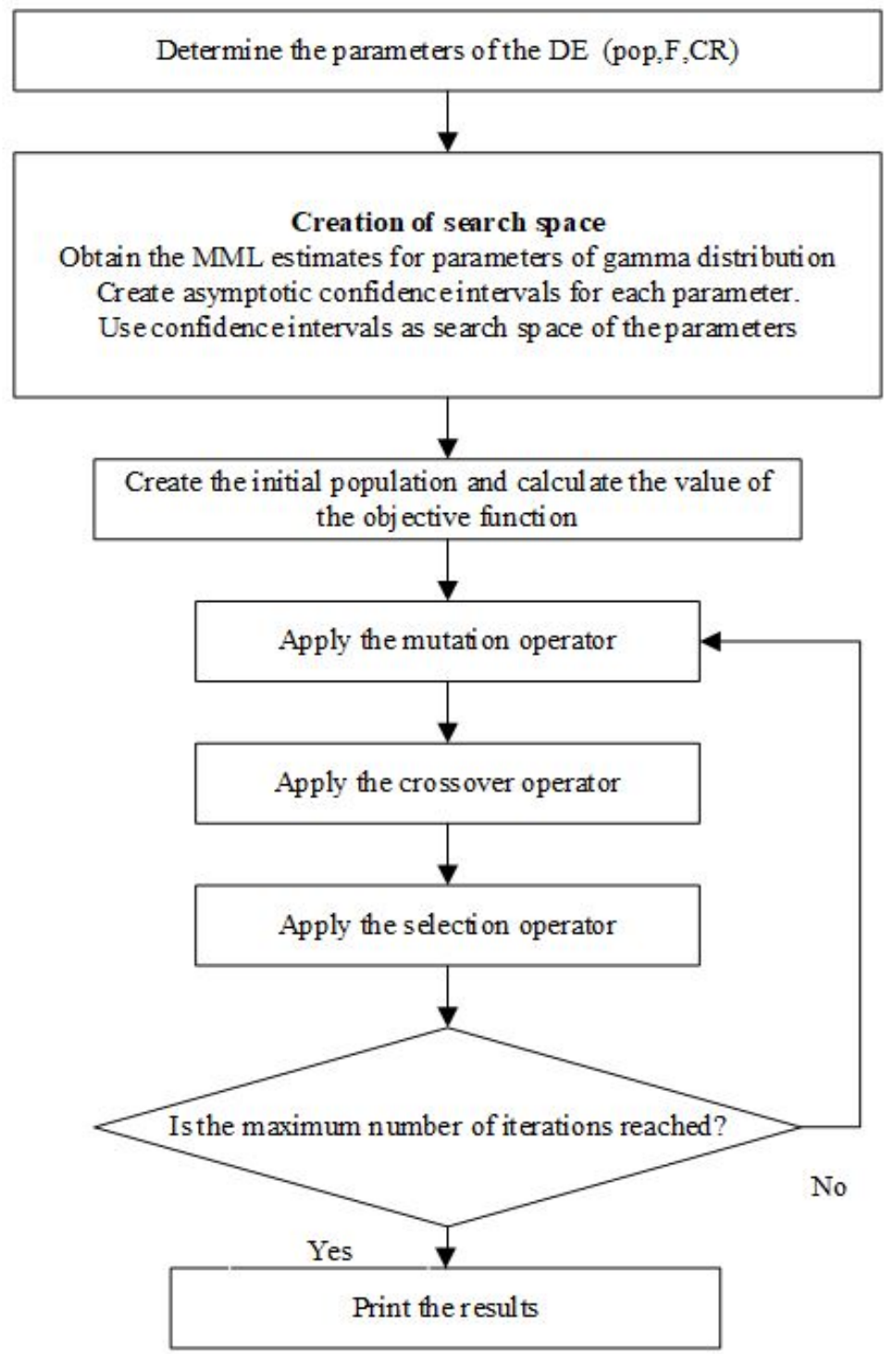

Figure 1. Flowchart for the proposed DE algorithm approach

Table 1. Search spaces for differential evolution algorithm.

\begin{tabular}{|l|l|}
\hline $\begin{array}{l}\text { Proposed Search Space (SSP_ML and } \\
\text { SSP_LS): }\end{array}$ & $95 \%$ confidence interval based on the MML estimates \\
\hline Search Space 1 (SS1_ML and SS1_LS): & {$[0,20]$ for $\sigma,[0,20]$ for $\beta,[-10,10]$ for $\mu$} \\
\hline Search Space 2 (SS2_ML and SS2_LS): & {$[0,100]$ for $\sigma,[0,100]$ for $\beta,[-50,50]$ for $\mu$} \\
\hline
\end{tabular}

when all cases are considered. Both the ML and LS estimates of the parameters for the 3-p Gamma distribution are calculated using the DE algorithm. The simulated mean, Mean Square Error (MSE) and deficiency (DEF) values are given in Tables 2-10. The $\mathrm{DEF}$ values are calculated as the sum of the MSE as follows:

$$
\operatorname{DEF}(\hat{\sigma}, \hat{\beta}, \hat{\mu})=\operatorname{MSE}(\hat{\sigma})+M S E(\hat{\beta})+M S E(\hat{\mu})
$$


Table 2. Simulation results for the estimation of the parameters $\theta=(3,1,-2)$ for $p o p=10$.

\begin{tabular}{|c|c|c|c|c|c|c|c|c|}
\hline \multirow[b]{2}{*}{$\mathrm{n}$} & \multirow[b]{2}{*}{ Method } & \multicolumn{2}{|c|}{$\hat{\sigma}$} & \multicolumn{2}{|c|}{$\beta$} & \multicolumn{2}{|c|}{$\hat{\mu}$} & \multirow[b]{2}{*}{ DEF } \\
\hline & & Mean & MSE & Mean & MSE & Mean & MSE & \\
\hline \multirow[t]{6}{*}{10} & $\mathrm{SSP}_{\mathrm{S}} \mathrm{ML}$ & 4.1081 & 6.3603 & 0.8273 & 0.1188 & -1.9823 & 0.7847 & 7.2639 \\
\hline & SS1_ML & 2.9587 & 13.4602 & 1.9513 & 2.7452 & -1.7448 & 1.9343 & 18.1397 \\
\hline & $\mathrm{SS} 2 \_\mathrm{ML}$ & 10.2772 & 296.1017 & 2.1231 & 3.9120 & -9.9916 & 233.4482 & 533.462 \\
\hline & $\mathrm{SSP} \_\mathrm{LS}$ & 4.9978 & 8.1338 & 0.8744 & 0.0855 & -3.0801 & 2.9397 & 11.1590 \\
\hline & SS1_LS & 4.7277 & 20.6180 & 1.4697 & 1.1587 & -3.2869 & 6.5435 & 28.3202 \\
\hline & SS2_LS & 13.1472 & 528.8568 & 5.3684 & 82.8275 & -16.4342 & 451.4205 & 1063.1049 \\
\hline \multirow[t]{6}{*}{30} & SSP_ML & 3.1466 & 0.8031 & 0.9679 & 0.0333 & -1.9414 & 0.1927 & 1.02916 \\
\hline & SS1_ML & 3.4958 & 9.9920 & 1.2642 & 0.4509 & -2.1178 & 1.3599 & 11.8028 \\
\hline & $\mathrm{SS} 2 \_\mathrm{ML}$ & 10.6030 & 300.9108 & 1.7162 & 2.3138 & -10.1954 & 245.0608 & 548.2855 \\
\hline & SSP_LS & 3.5873 & 1.2773 & 0.9647 & 0.0307 & -2.3604 & 0.5391 & 1.8470 \\
\hline & $\mathrm{SS} 1 \_\mathrm{LS}$ & 4.5447 & 18.5068 & 1.3150 & 0.6667 & -3.0188 & 4.9930 & 24.1665 \\
\hline & SS2_LS & 10.5735 & 310.4276 & 4.5812 & 41.1483 & -18.2958 & 539.2399 & 890.8158 \\
\hline \multirow[t]{6}{*}{50} & $\mathrm{SSP} \_\mathrm{ML}$ & 2.8464 & 0.4929 & 1.0392 & 0.0279 & -1.8731 & 0.1243 & 0.6452 \\
\hline & $\mathrm{SS} 1 \_\mathrm{ML}$ & 3.6563 & 9.3872 & 1.1394 & 0.2531 & -2.2046 & 1.2856 & 10.9259 \\
\hline & SS2_ML & 10.5346 & 294.4134 & 1.7056 & 3.3944 & -10.0279 & 230.6749 & 528.4827 \\
\hline & $\mathrm{SSP} \_\mathrm{LS}$ & 3.1328 & 0.5080 & 1.0199 & 0.0217 & -2.1103 & 0.2035 & 0.7331 \\
\hline & SS1_LS & 4.9827 & 22.8831 & 1.2133 & 0.4902 & -3.1541 & 5.6937 & 29.0670 \\
\hline & SS2_LS & 12.9022 & 507.8048 & 4.4482 & 52.1437 & -15.4235 & 403.6760 & 963.6245 \\
\hline \multirow[t]{6}{*}{100} & $\mathrm{SSP} \_\mathrm{ML}$ & 2.8215 & 0.2478 & 1.0430 & 0.01457 & -1.9027 & 0.0619 & 0.3244 \\
\hline & $\mathrm{SS} 1 \_\mathrm{ML}$ & 3.5953 & 7.9865 & 1.1034 & 0.1841 & -2.2270 & 1.2396 & 9.4103 \\
\hline & SS2_ML & 10.4232 & 285.6879 & 1.6719 & 3.3319 & -10.4079 & 245.7482 & 534.768 \\
\hline & $\mathrm{SSP} \_\mathrm{LS}$ & 2.9650 & 0.2430 & 1.0336 & 0.0106 & -2.0246 & 0.1106 & 0.3642 \\
\hline & SS1_LS & 5.0581 & 22.0390 & 1.1738 & 0.4621 & -3.3092 & 6.0832 & 28.5843 \\
\hline & SS2_LS & 12.4918 & 468.1017 & 4.6259 & 57.5204 & -15.2758 & 407.4964 & 933.1186 \\
\hline \multirow[t]{6}{*}{250} & $\mathrm{SSP} \_\mathrm{ML}$ & 2.8218 & 0.1047 & 1.0462 & 0.0067 & -1.9367 & 0.0205 & 0.1320 \\
\hline & SS1_ML & 3.6613 & 6.8480 & 1.0542 & 0.1115 & -2.2907 & 1.0608 & 8.0204 \\
\hline & $\mathrm{SS} 2 \_\mathrm{ML}$ & 10.9778 & 285.3779 & 1.5901 & 5.3516 & -10.7245 & 251.501 & 542.2306 \\
\hline & $\mathrm{SSP} \_\mathrm{LS}$ & 2.8453 & 0.1041 & 1.0480 & 0.0063 & -1.9614 & 0.0376 & 0.1480 \\
\hline & SS1_LS & 4.8449 & 19.0609 & 1.1654 & 0.4484 & -3.1308 & 4.7909 & 24.3002 \\
\hline & SS2_LS & 10.6644 & 318.6986 & 4.5701 & 47.9942 & -17.8700 & 520.3152 & 887.0080 \\
\hline \multirow[t]{6}{*}{500} & $\mathrm{SSP} \_\mathrm{ML}$ & 2.8825 & 0.0503 & 1.0290 & 0.0029 & -1.9559 & 0.0102 & 0.0634 \\
\hline & $\mathrm{SS} 1 \_\mathrm{ML}$ & 3.5845 & 6.3440 & 1.0549 & 0.1533 & -2.2392 & 0.8191 & 7.3166 \\
\hline & $\mathrm{SS} 2 \_\mathrm{ML}$ & 9.8410 & 481.4533 & 1.6262 & 3.0355 & -10.4271 & 246.2865 & 481.4533 \\
\hline & $\mathrm{SSP} \_\mathrm{LS}$ & 2.8758 & 0.0558 & 1.0327 & 0.0030 & -1.9561 & 0.0192 & 0.0780 \\
\hline & SS1_LS & 4.8133 & 19.5092 & 1.1741 & 0.3769 & -3.1835 & 5.1652 & 25.0513 \\
\hline & SS2_LS & 10.8120 & 332.7709 & 4.9663 & 73.5282 & -18.4075 & 529.6455 & 935.9445 \\
\hline \multirow[t]{6}{*}{1000} & $\mathrm{SSP} \_\mathrm{ML}$ & 2.9398 & 0.0242 & 1.0149 & 0.0013 & -1.9777 & 0.0044 & 0.0300 \\
\hline & SS1_ML & 3.5932 & 5.8670 & 1.0566 & 0.1903 & -2.2932 & 1.0644 & 7.1218 \\
\hline & $\mathrm{SS} 2 \_\mathrm{ML}$ & 10.8903 & 301.1794 & 1.6159 & 3.3527 & -11.0935 & 272.0797 & 576.6119 \\
\hline & SSP_LS & 2.9421 & 0.0282 & 1.0156 & 0.0012 & -1.9801 & 0.0104 & 0.0398 \\
\hline & SS1_LS & 5.1798 & 21.4202 & 1.1375 & 0.3950 & -3.3210 & 5.6725 & 27.4877 \\
\hline & SS2_LS & 11.4837 & 402.5591 & 4.5409 & 49.0188 & -17.7199 & 497.7178 & 949.2957 \\
\hline
\end{tabular}

The simulation results show that SSP and SS1 give better estimation values than SS2 in all cases. SS2 search space gives unreliable results because of very large. Moreover, SSP produces better results than SS1 in both the ML and LS methods according to the DEF criteria for most cases. As the number of sample size increased, a significant increase in the performance of the SSP search space has also observed. It is concluded that SSP is more suitable for estimating the parameters of the 3-p Gamma distribution except 
Table 3. Simulation results for the estimation of the parameters $\theta=(3,1,-2)$ for $p o p=25$.

\begin{tabular}{|c|c|c|c|c|c|c|c|c|}
\hline \multirow[b]{2}{*}{$\mathrm{n}$} & \multirow[b]{2}{*}{ Method } & \multicolumn{2}{|c|}{$\hat{\sigma}$} & \multicolumn{2}{|c|}{$\hat{\beta}$} & \multicolumn{2}{|r|}{$\hat{\mu}$} & \multirow[b]{2}{*}{ DEF } \\
\hline & & Mean & MSE & Mean & MSE & Mean & MSE & \\
\hline \multirow[t]{6}{*}{10} & SSP_ML & 3.8700 & 7.2861 & 0.8814 & 0.1202 & -1.8610 & 0.8559 & 8.2622 \\
\hline & SS1_ML & 2.1969 & 11.6152 & 2.4064 & 3.7625 & -1.3813 & 1.2610 & 16.6388 \\
\hline & $\mathrm{SS} 2 \_\mathrm{ML}$ & 3.4367 & 73.7615 & 2.3816 & 3.5016 & -2.2581 & 15.281 & 92.5445 \\
\hline & SSP_LS & 4.8712 & 8.9178 & 0.9023 & 0.1034 & -2.9515 & 2.7041 & 11.7253 \\
\hline & $\mathrm{SS} 1 \_\mathrm{LS}$ & 3.8317 & 15.1783 & 1.5499 & 1.6589 & -2.3889 & 3.0184 & 19.8557 \\
\hline & $\mathrm{SS} 2 \_\mathrm{LS}$ & 8.1162 & 306.1603 & 3.7872 & 37.8444 & -6.8014 & 130.0211 & 474.0258 \\
\hline \multirow[t]{6}{*}{30} & $\mathrm{SSP} \_\mathrm{ML}$ & 3.0062 & 0.8182 & 0.9973 & 0.0352 & -1.8827 & 0.1956 & 1.0490 \\
\hline & SS1_ML & 2.8752 & 5.7905 & 1.3248 & 0.4839 & -1.8127 & 0.5362 & 6.8106 \\
\hline & $\mathrm{SS} 2 \_\mathrm{ML}$ & 4.4656 & 88.1826 & 1.4980 & 0.7484 & -2.8087 & 21.236 & 110.1678 \\
\hline & SSP_LS & 3.5372 & 1.4636 & 0.9762 & 0.0375 & -2.3149 & 0.5231 & 2.0242 \\
\hline & $\mathrm{SS} 1 \_\mathrm{LS}$ & 3.9305 & 12.5226 & 1.1817 & 0.3005 & -2.3609 & 1.9107 & 14.7339 \\
\hline & $\mathrm{SS} 2 \_\mathrm{LS}$ & 7.9032 & 240.2697 & 3.3291 & 21.8602 & -8.8312 & 195.4372 & 457.5670 \\
\hline \multirow[t]{6}{*}{50} & $\mathrm{SSP} \_\mathrm{ML}$ & 2.7374 & 0.5794 & 1.0685 & 0.0348 & -1.8285 & 0.1363 & 0.7506 \\
\hline & SS1_ML & 2.8597 & 2.2978 & 1.1620 & 0.1810 & -1.8561 & 0.2551 & 2.7340 \\
\hline & $\mathrm{SS} 2 \_\mathrm{ML}$ & 4.7705 & 82.6319 & 1.3311 & 0.4161 & -2.9714 & 19.716 & 102.7642 \\
\hline & SSP_LS & 3.0971 & 0.6096 & 1.0294 & 0.0282 & -2.0775 & 0.2051 & 0.8429 \\
\hline & $\mathrm{SS} 1 \_\mathrm{LS}$ & 3.7560 & 10.4265 & 1.1414 & 0.2191 & -2.2635 & 1.4418 & 12.0874 \\
\hline & $\mathrm{SS} 2 \_\mathrm{LS}$ & 8.7670 & 297.4878 & 2.6827 & 17.7751 & -6.9580 & 132.6581 & 447.9209 \\
\hline \multirow[t]{6}{*}{100} & $\mathrm{SSP} \_\mathrm{ML}$ & 2.7481 & 0.2872 & 1.0616 & 0.0188 & -1.8734 & 0.0650 & 0.3710 \\
\hline & $\mathrm{SS} 1 \_\mathrm{ML}$ & 2.8744 & 1.2812 & 1.0826 & 0.0651 & -1.9029 & 0.1257 & 1.4721 \\
\hline & $\mathrm{SS} 2 \_\mathrm{ML}$ & 4.2958 & 66.2131 & 1.2218 & 0.2086 & -2.7249 & 15.6844 & 82.1062 \\
\hline & $\mathrm{SSP} \_\mathrm{LS}$ & 2.9168 & 0.2829 & 1.0432 & 0.0149 & -1.9899 & 0.1053 & 0.4032 \\
\hline & $\mathrm{SS} 1 \_\mathrm{LS}$ & 3.9077 & 10.3111 & 1.0583 & 0.1248 & -2.3261 & 1.3128 & 11.7487 \\
\hline & $\mathrm{SS} 2 \_\mathrm{LS}$ & 8.8412 & 317.7574 & 2.7961 & 24.0806 & -6.5267 & 107.2595 & 449.0975 \\
\hline \multirow[t]{6}{*}{250} & $\mathrm{SSP} \_\mathrm{ML}$ & 2.8023 & 0.1177 & 1.0514 & 0.0079 & -1.9292 & 0.0214 & 0.1471 \\
\hline & SS1_ML & 2.8975 & 0.4320 & 1.0485 & 0.0263 & -1.9525 & 0.0446 & 0.5030 \\
\hline & SS2_ML & 4.2272 & 58.8406 & 1.1664 & 0.1259 & -2.8007 & 16.7065 & 75.6786 \\
\hline & SSP_LS & 2.8301 & 0.1250 & 1.0500 & 0.0076 & -1.9475 & 0.0388 & 0.1714 \\
\hline & $\mathrm{SS} 1 \_\mathrm{LS}$ & 3.8379 & 9.0928 & 1.0346 & 0.0893 & -2.3108 & 1.1798 & 10.3619 \\
\hline & $\mathrm{SS} 2 \_\mathrm{LS}$ & 8.9133 & 306.1155 & 3.1061 & 28.2233 & -8.3519 & 172.9180 & 507.2569 \\
\hline \multirow[t]{6}{*}{500} & $\mathrm{SSP} \_\mathrm{ML}$ & 2.8726 & 0.0577 & 1.0315 & 0.0033 & -1.952 & 0.01093 & 0.07206 \\
\hline & SS1_ML & 2.9328 & 0.3180 & 1.0319 & 0.0152 & -1.9692 & 0.03217 & 0.3654 \\
\hline & $\mathrm{SS} 2 \_\mathrm{ML}$ & 4.3728 & 65.1725 & 1.1248 & 0.1014 & -2.7968 & 15.1136 & 80.3876 \\
\hline & $\mathrm{SSP} \_\mathrm{LS}$ & 2.8743 & 0.0630 & 1.0328 & 0.0035 & -1.9529 & 0.0196 & 0.0861 \\
\hline & $\mathrm{SS} 1 \_\mathrm{LS}$ & 3.9218 & 9.9909 & 1.0106 & 0.0727 & -2.3333 & 1.1863 & 11.2498 \\
\hline & $\mathrm{SS} 2 \_\mathrm{LS}$ & 9.6920 & 335.7865 & 2.6505 & 11.6950 & -9.0366 & 197.3591 & 544.8407 \\
\hline \multirow[t]{6}{*}{1000} & $\mathrm{SSP} \_\mathrm{ML}$ & 2.9329 & 0.0263 & 1.0165 & 0.0014 & -1.9747 & 0.0045 & 0.0323 \\
\hline & $\mathrm{SS} 1 \_\mathrm{ML}$ & 2.9586 & 0.2512 & 1.0225 & 0.0094 & -1.9849 & 0.0283 & 0.2890 \\
\hline & $\mathrm{SS} 2 \_\mathrm{ML}$ & 4.1003 & 45.1303 & 1.1123 & 0.08952 & -2.7366 & 13.6658 & 58.8857 \\
\hline & SSP_LS & 2.9375 & 0.0327 & 1.0158 & 0.0015 & -1.9753 & 0.0105 & 0.0447 \\
\hline & $\mathrm{SS} 1 \_\mathrm{LS}$ & 4.0281 & 9.4578 & 0.9909 & 0.0728 & -2.3915 & 1.1256 & 10.6562 \\
\hline & $\mathrm{SS} 2 \_\mathrm{LS}$ & 9.6719 & 337.4216 & 2.6890 & 10.6083 & -9.4090 & 200.6948 & 548.7248 \\
\hline
\end{tabular}

for a few cases, for example $\theta=(4,0.5,10), n=10$, pop $=25$ and 50 for the LS and $\theta=(2.56,0.75,8), n=10, p o p=25$ for the ML and LS. Therefore, it can be said that the proposed DE approach is preferable than the others. 
Table 4. Simulation results for the estimation of the parameters $\theta=(3,1,-2)$ for $p o p=50$.

\begin{tabular}{|c|c|c|c|c|c|c|c|c|}
\hline & & & & & & & $\hat{\mu}$ & \\
\hline $\mathrm{n}$ & Method & Mean & MSE & Mean & MSE & Mean & MSE & $\mathrm{DEF}$ \\
\hline \multirow[t]{6}{*}{10} & $\mathrm{SSP}_{\mathrm{ALL}}$ & 3.8313 & 7.8802 & 0.9015 & 0.1225 & -1.8383 & 0.9051 & 8.9079 \\
\hline & $\mathrm{SS} 1 \_\mathrm{ML}$ & 2.1334 & 12.4083 & 2.5724 & 4.3032 & -1.3570 & 1.3122 & 18.0238 \\
\hline & $\mathrm{SS} 2 \_\mathrm{ML}$ & 1.8889 & 25.7838 & 2.6660 & 4.3152 & -1.3236 & 1.8228 & 31.9219 \\
\hline & SSP_LS & 4.9744 & 10.3985 & 0.9064 & 0.1129 & -2.9611 & 2.8361 & 13.3474 \\
\hline & $\mathrm{SS} 1 \_\mathrm{LS}$ & 3.8160 & 14.0347 & 1.5306 & 1.5668 & -2.3218 & 2.5635 & 18.1650 \\
\hline & $\mathrm{SS} 2 \_\mathrm{LS}$ & 6.2842 & 270.3826 & 3.5075 & 39.5510 & -3.4243 & 42.3600 & 352.2937 \\
\hline \multirow[t]{6}{*}{30} & $\mathrm{SSP} \_\mathrm{ML}$ & 2.9827 & 0.8296 & 1.0037 & 0.0365 & -1.8740 & 0.1959 & 1.0622 \\
\hline & SS1_ML & 2.9501 & 6.3661 & 1.3269 & 0.5097 & -1.8234 & 0.5480 & 7.4239 \\
\hline & $\mathrm{SS} 2 \_\mathrm{ML}$ & 2.6886 & 13.7491 & 1.4812 & 0.6489 & -1.8020 & 1.3787 & 15.7768 \\
\hline & SSP_LS & 3.5093 & 1.5774 & 0.9852 & 0.0418 & -2.2947 & 0.5237 & 2.1429 \\
\hline & $\mathrm{SS} 1 \_\mathrm{LS}$ & 3.7228 & 11.4252 & 1.1991 & 0.3329 & -2.2041 & 1.2908 & 13.0490 \\
\hline & $\mathrm{SS} 2 \_\mathrm{LS}$ & 6.3778 & 215.3560 & 2.4797 & 8.1826 & -4.1700 & 51.4928 & 275.0314 \\
\hline \multirow[t]{6}{*}{50} & $\mathrm{SSP} \_\mathrm{ML}$ & 2.7237 & 0.6010 & 1.0734 & 0.0362 & -1.8239 & 0.1404 & 0.7777 \\
\hline & $\mathrm{SS} 1 \_\mathrm{ML}$ & 2.9369 & 3.1182 & 1.1523 & 0.1777 & -1.8685 & 0.2878 & 3.5838 \\
\hline & $\mathrm{SS} 2 \_\mathrm{ML}$ & 2.9647 & 17.7667 & 1.2830 & 0.2768 & -1.9249 & 1.3972 & 19.4408 \\
\hline & SSP_LS & 3.0960 & 0.6808 & 1.0342 & 0.0323 & -2.0742 & 0.2098 & 0.9229 \\
\hline & $\mathrm{SS} 1 \_\mathrm{LS}$ & 3.5481 & 8.3221 & 1.1285 & 0.1844 & -2.1361 & 0.8902 & 9.3967 \\
\hline & $\mathrm{SS} 2 \_\mathrm{LS}$ & 5.7246 & 189.0630 & 2.1140 & 6.0979 & -3.4530 & 39.9505 & 235.1114 \\
\hline \multirow[t]{6}{*}{100} & SSP_ML & 2.7385 & 0.2973 & 1.0647 & 0.0196 & -1.8702 & 0.0665 & 0.3835 \\
\hline & SS1_ML & 2.9109 & 1.1020 & 1.0716 & 0.0635 & -1.9112 & 0.1157 & 1.2813 \\
\hline & $\mathrm{SS} 2 \_\mathrm{ML}$ & 3.0300 & 14.8081 & 1.1771 & 0.1315 & -1.9786 & 1.3586 & 16.2983 \\
\hline & SSP_LS & 2.9116 & 0.3159 & 1.0463 & 0.0170 & -1.9853 & 0.1098 & 0.4427 \\
\hline & $\mathrm{SS} 1 \_\mathrm{LS}$ & 3.3758 & 5.7175 & 1.0838 & 0.1075 & -2.0881 & 0.6062 & 6.4312 \\
\hline & $\mathrm{SS} 2 \_\mathrm{LS}$ & 6.6015 & 253.7575 & 2.0297 & 5.3945 & -3.6425 & 35.3657 & 294.5177 \\
\hline \multirow[t]{6}{*}{250} & $\mathrm{SSP} \_\mathrm{ML}$ & 2.7993 & 0.1199 & 1.0523 & 0.0081 & -1.9283 & 0.0215 & 0.1496 \\
\hline & $\mathrm{SS} 1 \_\mathrm{ML}$ & 2.9006 & 0.3569 & 1.0398 & 0.0216 & -1.9505 & 0.0328 & 0.3569 \\
\hline & $\mathrm{SS} 2 \_\mathrm{ML}$ & 2.9673 & 12.9489 & 1.1166 & 0.0587 & -1.9987 & 1.1020 & 14.1097 \\
\hline & SSP_LS & 2.8270 & 0.1342 & 1.0514 & 0.0084 & -1.9458 & 0.0395 & 0.1821 \\
\hline & $\mathrm{SS} 1 \_\mathrm{LS}$ & 3.3329 & 3.9782 & 1.0389 & 0.0561 & -2.0954 & 0.4253 & 4.4597 \\
\hline & $\mathrm{SS} 2 \_\mathrm{LS}$ & 7.3622 & 259.7597 & 2.0636 & 7.4142 & -4.8842 & 64.1653 & 331.3391 \\
\hline \multirow[t]{6}{*}{500} & $\mathrm{SSP} \_\mathrm{ML}$ & 2.8716 & 0.0583 & 1.0319 & 0.0034 & -1.9517 & 0.0109 & 0.0728 \\
\hline & $\mathrm{SS} 1 \_\mathrm{ML}$ & 2.9383 & 0.1532 & 1.0220 & 0.0106 & -1.9664 & 0.0175 & 0.1814 \\
\hline & $\mathrm{SS} 2 \_\mathrm{ML}$ & 2.8774 & 2.7291 & 1.1009 & 0.0482 & -1.9938 & 0.7305 & 3.5079 \\
\hline & $\mathrm{SSP} \_\mathrm{LS}$ & 2.8762 & 0.0690 & 1.0327 & 0.0038 & -1.9534 & 0.0206 & 0.0934 \\
\hline & $\mathrm{SS} 1 \_\mathrm{LS}$ & 3.2780 & 2.5897 & 1.0209 & 0.0385 & -2.0835 & 0.3227 & 2.9509 \\
\hline & $\mathrm{SS} 2 \_\mathrm{LS}$ & 7.7825 & 289.9620 & 2.1047 & 7.0621 & -5.1289 & 75.5625 & 372.5865 \\
\hline \multirow[t]{6}{*}{1000} & $\mathrm{SSP} \_\mathrm{ML}$ & 2.9316 & 0.02677 & 1.0169 & 0.0015 & -1.9743 & 0.0045 & 0.0328 \\
\hline & $\mathrm{SS} 1 \_\mathrm{ML}$ & 2.9559 & 0.0862 & 1.0145 & 0.0054 & -1.9788 & 0.0088 & 0.1005 \\
\hline & SS2_ML & 3.0109 & 6.9185 & 1.0835 & 0.0310 & -2.0760 & 2.8762 & 9.8258 \\
\hline & SSP_LS & 2.9351 & 0.0359 & 1.0166 & 0.0017 & -1.9743 & 0.0107 & 0.0483 \\
\hline & SS1_LS & 3.3312 & 2.8849 & 1.0073 & 0.0322 & -2.1094 & 0.3209 & 3.2381 \\
\hline & $\mathrm{SS} 2 \_\mathrm{LS}$ & 7.0331 & 241.5506 & 2.1044 & 6.2384 & -4.7036 & 59.3074 & 307.0964 \\
\hline
\end{tabular}


Table 5. Simulation results for the estimation of the parameters $\theta=(4,0.5,10)$ for $p o p=10$.

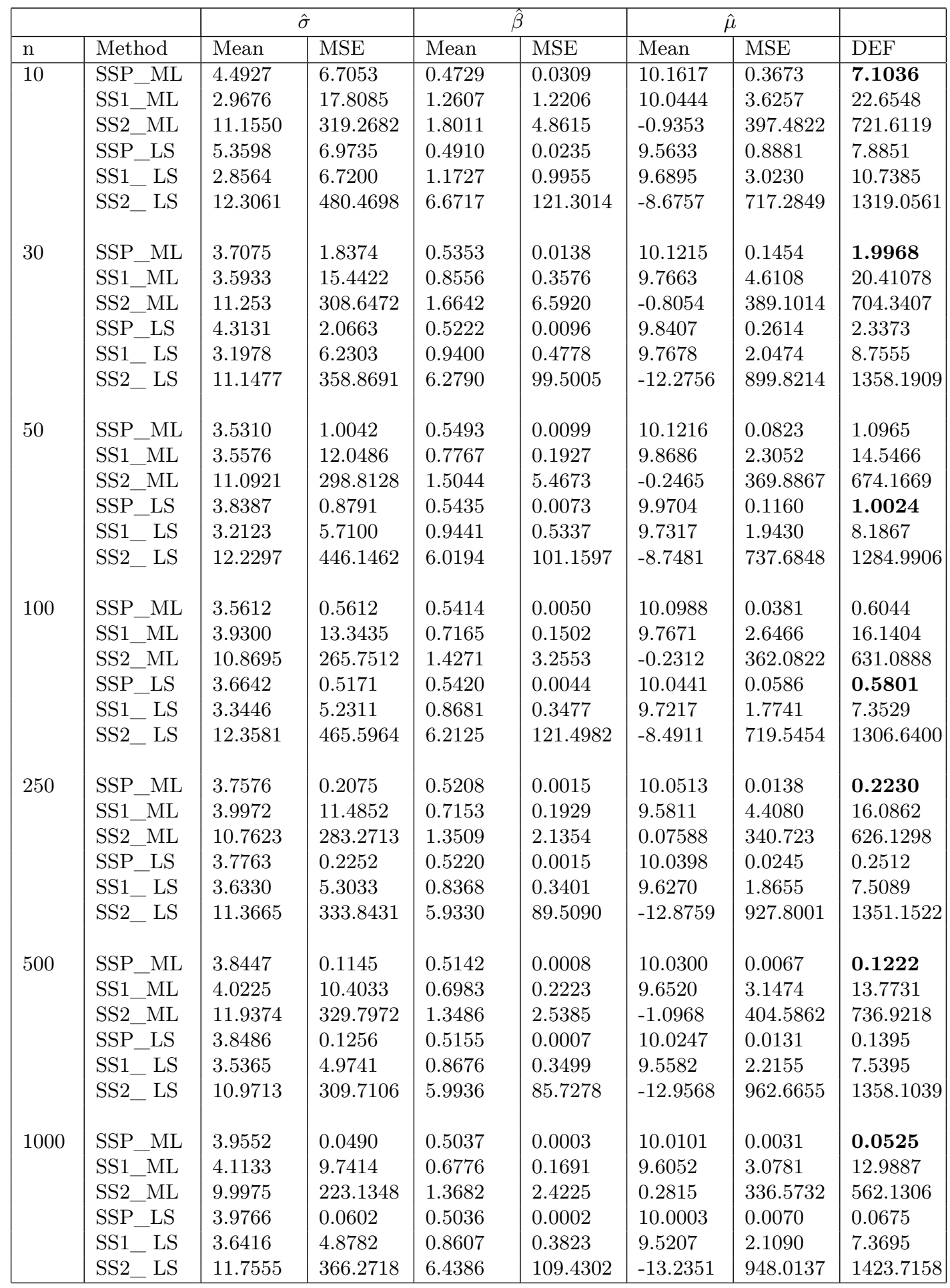


Table 6. Simulation results for the estimation of the parameters $\theta=(4,0.5,10)$ for $p o p=25$.

\begin{tabular}{|c|c|c|c|c|c|c|c|c|}
\hline & & & & & & & $\hat{\mu}$ & \\
\hline $\mathrm{n}$ & Method & Mean & MSE & Mean & MSE & Mean & MSE & DEF \\
\hline \multirow[t]{6}{*}{10} & $\mathrm{SSP}_{\mathrm{S}} \mathrm{ML}$ & 4.3236 & 8.4890 & 0.5014 & 0.036 & 10.2192 & 0.4208 & 8.9463 \\
\hline & $\mathrm{SS} 1 \_\mathrm{ML}$ & 1.8934 & 12.0569 & 1.3959 & 1.3241 & 10.5883 & 0.6502 & 14.0313 \\
\hline & $\mathrm{SS} 2 \_\mathrm{ML}$ & 3.2666 & 75.1719 & 1.5312 & 1.5971 & 9.4402 & 29.0729 & 105.8421 \\
\hline & SSP_LS & 5.3234 & 8.0966 & 0.5010 & 0.0308 & 9.6205 & 0.8489 & 8.9764 \\
\hline & $\mathrm{SS} 1 \_\mathrm{LS}$ & 2.6049 & 5.9594 & 1.0289 & 0.7892 & 10.2932 & 0.5848 & 7.3333 \\
\hline & $\mathrm{SS} 2 \_\mathrm{LS}$ & 8.5574 & 333.5715 & 5.5630 & 119.0918 & 2.4118 & 263.4666 & 716.1299 \\
\hline \multirow[t]{6}{*}{30} & $\mathrm{SSP} \_\mathrm{ML}$ & 3.4952 & 2.1289 & 0.5595 & 0.0190 & 10.1714 & 0.1568 & 2.3048 \\
\hline & SS1_ML & 2.9972 & 6.6971 & 0.7622 & 0.1864 & 10.2651 & 0.2802 & 7.1638 \\
\hline & $\mathrm{SS} 2 \_\mathrm{ML}$ & 4.1640 & 78.4705 & 1.0280 & 0.5166 & 9.1412 & 28.4627 & 107.4544 \\
\hline & SSP_LS & 4.1747 & 2.1961 & 0.5310 & 0.0134 & 9.9012 & 0.2386 & 2.4480 \\
\hline & $\mathrm{SS} 1 \_\mathrm{LS}$ & 2.7366 & 4.5384 & 0.7907 & 0.1807 & 10.2745 & 0.3122 & 5.0313 \\
\hline & $\mathrm{SS} 2 \_\mathrm{LS}$ & 8.8231 & 280.2917 & 4.7460 & 74.0181 & -0.8255 & 375.4819 & 729.7918 \\
\hline \multirow[t]{6}{*}{50} & $\mathrm{SSP} \_\mathrm{ML}$ & 3.3617 & 1.2959 & 0.5689 & 0.0140 & 10.1615 & 0.0956 & 1.4057 \\
\hline & SS1_ML & 3.0441 & 4.7673 & 0.6879 & 0.0883 & 10.2237 & 0.1930 & 5.04875 \\
\hline & SS2_ML & 4.6207 & 89.5504 & 0.9251 & 0.3488 & 9.0328 & 27.4662 & 117.3655 \\
\hline & SSP_LS & 3.7785 & 1.0971 & 0.5480 & 0.0096 & 10.0017 & 0.1240 & 1.2306 \\
\hline & $\mathrm{SS} 1 \_\mathrm{LS}$ & 2.9883 & 3.8932 & 0.7219 & 0.1146 & 10.2106 & 0.2675 & 4.2753 \\
\hline & SS2_LS & 8.8556 & 338.8390 & 4.2220 & 75.1658 & 2.4458 & 260.7591 & 674.7639 \\
\hline \multirow[t]{6}{*}{100} & SSP_ML & 3.5018 & 0.7172 & 0.5491 & 0.0064 & 10.1111 & 0.0422 & 0.7172 \\
\hline & $\mathrm{SS} 1 \_\mathrm{ML}$ & 3.2427 & 2.9336 & 0.6238 & 0.0388 & 10.1610 & 0.1059 & 3.0784 \\
\hline & SS2_ML & 4.5396 & 85.4658 & 0.8147 & 0.2051 & 9.1501 & 24.4157 & 110.0868 \\
\hline & SSP_LS & 3.6452 & 0.5737 & 0.5424 & 0.0052 & 10.0578 & 0.0579 & 0.6369 \\
\hline & $\mathrm{SS} 1 \_\mathrm{LS}$ & 3.0900 & 3.1419 & 0.6774 & 0.0741 & 10.1776 & 0.1891 & 3.4051 \\
\hline & $\mathrm{SS} 2 \_\mathrm{LS}$ & 8.9699 & 329.9158 & 3.8824 & 43.5022 & 2.7742 & 227.4570 & 600.8750 \\
\hline \multirow[t]{6}{*}{250} & $\mathrm{SSP} \_\mathrm{ML}$ & 3.7249 & 0.2483 & 0.5243 & 0.0020 & 10.0581 & 0.0152 & 0.2656 \\
\hline & $\mathrm{SS} 1 \_\mathrm{ML}$ & 3.3996 & 1.6394 & 0.5831 & 0.01836 & 10.1176 & 0.0573 & 1.7152 \\
\hline & $\mathrm{SS} 2 \_\mathrm{ML}$ & 4.6783 & 69.9571 & 0.7591 & 0.1640 & 8.9209 & 28.6090 & 98.7302 \\
\hline & SSP_LS & 3.7555 & 0.2492 & 0.5229 & 0.0017 & 10.0495 & 0.0242 & 0.2752 \\
\hline & $\mathrm{SS} 1 \_\mathrm{LS}$ & 3.3399 & 2.5821 & 0.6300 & 0.0482 & 10.1170 & 0.1309 & 2.7611 \\
\hline & $\mathrm{SS} 2 \_\mathrm{LS}$ & 9.6891 & 347.1285 & 4.5036 & 60.4417 & -0.1056 & 334.9611 & 742.5313 \\
\hline \multirow[t]{6}{*}{500} & $\mathrm{SSP} \_\mathrm{ML}$ & 3.8337 & 0.1268 & 0.5152 & 0.0009 & 10.0324 & 0.0072 & 0.1350 \\
\hline & $\mathrm{SS} 1 \_\mathrm{ML}$ & 3.4737 & 1.3998 & 0.5692 & 0.0116 & 10.0970 & 0.0463 & 1.4578 \\
\hline & $\mathrm{SS} 2 \_\mathrm{ML}$ & 5.0636 & 83.8007 & 0.7383 & 0.1671 & 8.7998 & 30.8414 & 114.8093 \\
\hline & $\mathrm{SSP} \_\mathrm{LS}$ & 3.8474 & 0.1332 & 0.5150 & 0.0008 & 10.0279 & 0.0127 & 0.1468 \\
\hline & $\mathrm{SS} 1 \_\mathrm{LS}$ & 3.4531 & 2.4743 & 0.6183 & 0.0423 & 10.0766 & 0.1374 & 2.6539 \\
\hline & $\mathrm{SS} 2 \_\mathrm{LS}$ & 10.1780 & 353.7534 & 4.1843 & 57.8931 & -1.0371 & 397.2929 & 808.9394 \\
\hline \multirow[t]{6}{*}{1000} & $\mathrm{SSP} \_\mathrm{ML}$ & 3.9431 & 0.0559 & 0.5048 & 0.0003 & 10.0124 & 0.0034 & 0.0597 \\
\hline & $\mathrm{SS} 1 \_\mathrm{ML}$ & 3.5021 & 0.7336 & 0.5578 & 0.0078 & 10.0886 & 0.0249 & 0.7664 \\
\hline & SS2_ML & 4.8574 & 69.9133 & 0.7310 & 0.1649 & 8.8523 & 29.7498 & 99.8281 \\
\hline & $\mathrm{SSP} \_\mathrm{LS}$ & 3.9699 & 0.0699 & 0.5039 & 0.0003 & 10.0035 & 0.0070 & 0.0772 \\
\hline & $\mathrm{SS} 1_{-} \mathrm{LS}$ & 3.5570 & 2.2414 & 0.6052 & 0.0399 & 10.0488 & 0.1247 & 2.4061 \\
\hline & $\mathrm{SS} 2 \_\mathrm{LS}$ & 10.2309 & 327.2825 & 3.9128 & 46.5160 & -1.7804 & 410.7385 & 784.5371 \\
\hline
\end{tabular}


Table 7. Simulation results for the estimation of the parameters $\theta=(4,0.5,10)$ for $p o p=50$.

\begin{tabular}{|c|c|c|c|c|c|c|c|c|}
\hline \multirow[b]{2}{*}{$\mathrm{n}$} & \multirow[b]{2}{*}{ Method } & \multicolumn{2}{|c|}{$\hat{\sigma}$} & \multicolumn{2}{|c|}{$\hat{\beta}$} & \multicolumn{2}{|c|}{$\hat{\mu}$} & \multirow[b]{2}{*}{ DEF } \\
\hline & & Mean & MSE & Mean & MSE & Mean & MSE & \\
\hline \multirow[t]{6}{*}{10} & SSP_ML & 4.2683 & 9.1652 & 0.5131 & 0.0395 & 10.2371 & 0.4414 & 9.6462 \\
\hline & SS1_ML & 2.0592 & 12.3012 & 1.3890 & 1.3435 & 10.5647 & 0.6424 & 14.2872 \\
\hline & $\mathrm{SS} 2 \_\mathrm{ML}$ & 1.5514 & 17.4401 & 1.5498 & 1.5544 & 10.5852 & 1.5447 & 20.5392 \\
\hline & SSP_LS & 5.3369 & 9.2060 & 0.5087 & 0.0355 & 9.6356 & 0.8885 & 10.1299 \\
\hline & $\mathrm{SS} 1 \_\mathrm{LS}$ & 2.7455 & 5.3813 & 0.9579 & 0.6875 & 10.2874 & 0.5161 & 6.5850 \\
\hline & $\mathrm{SS} 2 \_\mathrm{LS}$ & 5.1117 & 167.9172 & 4.5674 & 87.4712 & 7.5098 & 91.2558 & 346.6442 \\
\hline \multirow[t]{6}{*}{30} & SSP_ML & 3.4435 & 2.2750 & 0.5673 & 0.0210 & 10.1831 & 0.1639 & 2.4600 \\
\hline & SS1_ML & 3.1289 & 5.7201 & 0.7248 & 0.1661 & 10.2510 & 0.2466 & 6.1329 \\
\hline & $\mathrm{SS} 2 \_\mathrm{ML}$ & 2.8543 & 17.3835 & 0.9123 & 0.3210 & 10.1711 & 1.9764 & 19.6809 \\
\hline & SSP_LS & 4.1580 & 2.5269 & 0.5374 & 0.0159 & 9.9092 & 0.2488 & 2.7916 \\
\hline & $\mathrm{SS} 1_{-} \mathrm{LS}$ & 2.8942 & 3.9780 & 0.7349 & 0.1350 & 10.2719 & 0.2396 & 4.3526 \\
\hline & $\mathrm{SS} 2 \_\mathrm{LS}$ & 6.8806 & 251.1246 & 4.1015 & 65.9729 & 6.0875 & 108.5129 & 425.6103 \\
\hline \multirow[t]{6}{*}{50} & $\mathrm{SSP} \_\mathrm{ML}$ & 3.3449 & 1.3629 & 0.5731 & 0.0150 & 10.1667 & 0.0990 & 1.4770 \\
\hline & SS1_ML & 3.1075 & 3.3861 & 0.6546 & 0.0651 & 10.2183 & 0.1484 & 3.5997 \\
\hline & $\mathrm{SS} 2 \_\mathrm{ML}$ & 2.8689 & 13.2331 & 0.8136 & 0.1861 & 10.1837 & 0.9180 & 14.3374 \\
\hline & SSP_LS & 3.7505 & 1.2272 & 0.5530 & 0.0113 & 10.0114 & 0.1276 & 1.3662 \\
\hline & SS1_LS & 2.9810 & 3.4078 & 0.6900 & 0.0811 & 10.2375 & 0.1966 & 3.6856 \\
\hline & $\mathrm{SS} 2 \_\mathrm{LS}$ & 5.4397 & 166.3668 & 2.9944 & 26.5011 & 7.8219 & 66.6044 & 259.4723 \\
\hline \multirow[t]{6}{*}{100} & $\mathrm{SSP} \_\mathrm{ML}$ & 3.4897 & 0.6931 & 0.5507 & 0.0068 & 10.1134 & 0.0433 & 0.7433 \\
\hline & SS1_ML & 3.3902 & 2.0872 & 0.5885 & 0.0251 & 10.1443 & 0.08131 & 2.1936 \\
\hline & $\mathrm{SS} 2 \_\mathrm{ML}$ & 3.0705 & 12.1302 & 0.7413 & 0.1235 & 10.1258 & 1.0299 & 13.2837 \\
\hline & SSP_LS & 3.6350 & 0.6271 & 0.5443 & 0.0059 & 10.0620 & 0.0597 & 0.6927 \\
\hline & $\mathrm{SS} 1 \_\mathrm{LS}$ & 3.1200 & 2.4466 & 0.6346 & 0.0434 & 10.2113 & 0.1353 & 2.6253 \\
\hline & $\mathrm{SS} 2 \_\mathrm{LS}$ & 5.2318 & 151.6501 & 2.8139 & 24.9800 & 8.2256 & 47.3678 & 223.9978 \\
\hline \multirow[t]{6}{*}{250} & $\mathrm{SSP} \_\mathrm{ML}$ & 3.7187 & 0.2564 & 0.5250 & 0.0020 & 10.0593 & 0.0156 & 0.2742 \\
\hline & $\mathrm{SS} 1 \_\mathrm{ML}$ & 3.5323 & 1.0121 & 0.5555 & 0.0101 & 10.0989 & 0.0387 & 1.0610 \\
\hline & $\mathrm{SS} 2 \_\mathrm{ML}$ & 3.2166 & 6.2031 & 0.6667 & 0.06084 & 10.0771 & 0.6837 & 6.9477 \\
\hline & SSP_LS & 3.7413 & 0.2735 & 0.5244 & 0.0020 & 10.0533 & 0.0248 & 0.3003 \\
\hline & $\mathrm{SS} 1 \_\mathrm{LS}$ & 3.3449 & 2.0583 & 0.5943 & 0.0242 & 10.1601 & 0.0936 & 2.1761 \\
\hline & $\mathrm{SS} 2 \_\mathrm{LS}$ & 7.2647 & 242.9537 & 2.9801 & 22.7486 & 5.3469 & 142.4554 & 408.1577 \\
\hline \multirow[t]{6}{*}{500} & $\mathrm{SSP} \_\mathrm{ML}$ & 3.8315 & 0.1298 & 0.5155 & 0.0009 & 10.0328 & 0.0073 & 0.1381 \\
\hline & SS1_ML & 3.5890 & 0.6914 & 0.5472 & 0.0067 & 10.0791 & 0.0259 & 0.7241 \\
\hline & $\mathrm{SS} 2 \_\mathrm{ML}$ & 3.2630 & 4.9462 & 0.6421 & 0.04873 & 10.0961 & 0.3291 & 5.3241 \\
\hline & $\mathrm{SSP} \_\mathrm{LS}$ & 3.8459 & 0.1501 & 0.5155 & 0.0009 & 10.0280 & 0.0136 & 0.1647 \\
\hline & $\mathrm{SS} 1 \_\mathrm{LS}$ & 3.5045 & 1.7132 & 0.5734 & 0.0164 & 10.1136 & 0.0783 & 1.8079 \\
\hline & $\mathrm{SS} 2 \_\mathrm{LS}$ & 8.5492 & 337.4011 & 2.9457 & 27.7306 & 5.1378 & 139.5766 & 504.7083 \\
\hline \multirow[t]{6}{*}{1000} & $\mathrm{SSP} \_\mathrm{ML}$ & 3.9408 & 0.0570 & 0.5050 & 0.0003 & 10.0129 & 0.0034 & 0.0609 \\
\hline & SS1_ML & 3.6291 & 0.4379 & 0.5391 & 0.0040 & 10.0702 & 0.01640 & 0.4583 \\
\hline & $\mathrm{SS} 2 \_\mathrm{ML}$ & 3.2683 & 2.7705 & 0.6251 & 0.0391 & 10.1020 & 0.101541 & 2.7705 \\
\hline & SSP_LS & 3.9646 & 0.0768 & 0.5044 & 0.0004 & 10.0048 & 0.0072 & 0.0844 \\
\hline & $\mathrm{SS} 1 \_\mathrm{LS}$ & 3.5715 & 1.3889 & 0.5644 & 0.0133 & 10.0867 & 0.0628 & 1.4651 \\
\hline & $\mathrm{SS} 2 \_\mathrm{LS}$ & 7.5491 & 243.1289 & 3.0848 & 36.1980 & 5.3303 & 136.3073 & 415.6343 \\
\hline
\end{tabular}


Table 8. Simulation results for the estimation of the parameters $\theta=(2.56,0.75,8)$ for pop $=10$.

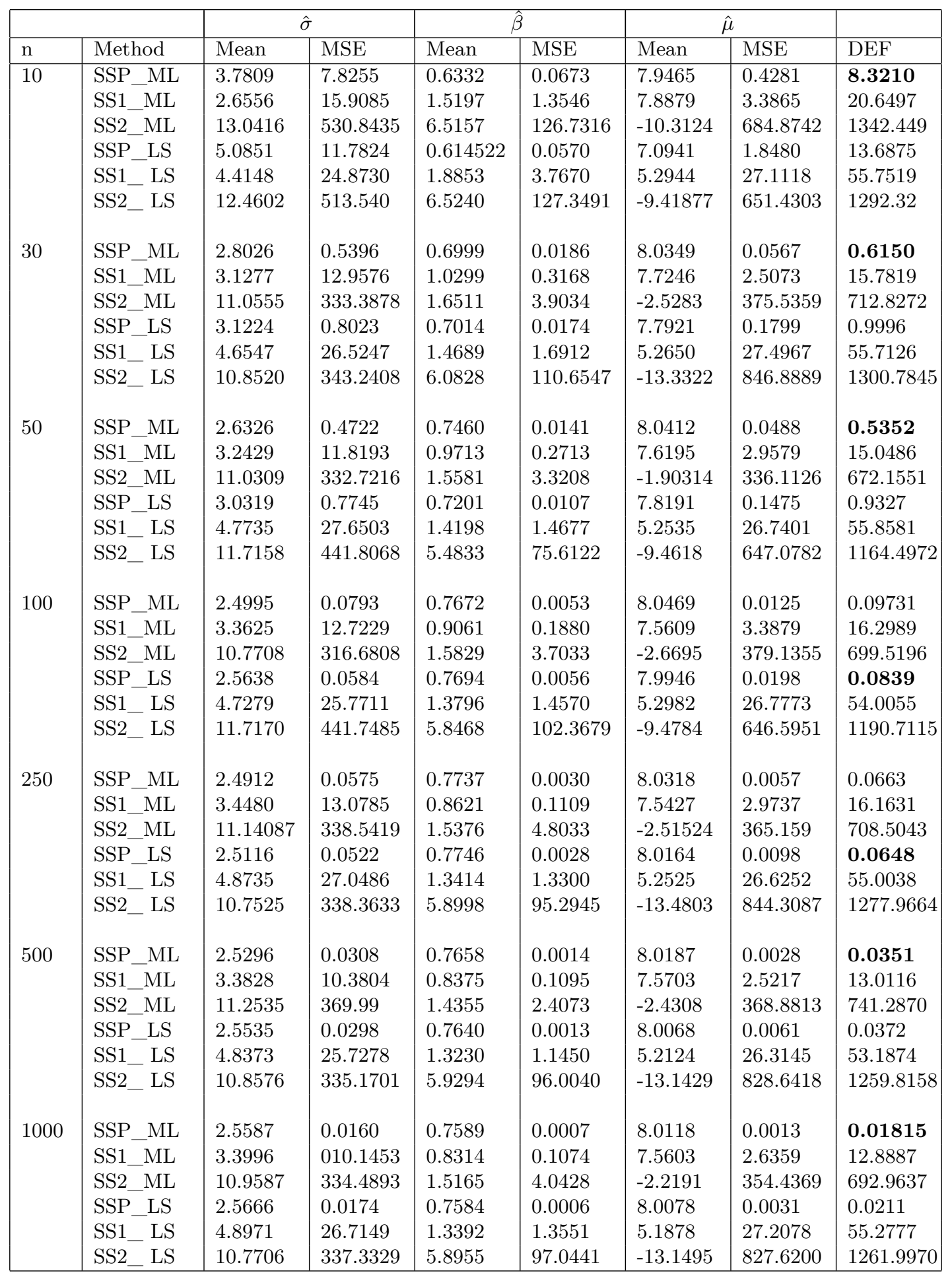


Table 9. Simulation results for the estimation of the parameters $\theta=(2.56,0.75,8)$ for pop $=25$.

\begin{tabular}{|c|c|c|c|c|c|c|c|c|}
\hline & & \multicolumn{2}{|c|}{$\hat{\sigma}$} & \multicolumn{2}{|c|}{$\hat{\beta}$} & \multicolumn{2}{|r|}{$\hat{\mu}$} & \\
\hline $\mathrm{n}$ & Method & Mean & MSE & Mean & MSE & Mean & MSE & DEF \\
\hline \multirow[t]{6}{*}{10} & SSP $\quad$ ML & 3.2213 & 7.8948 & 0.7138 & 0.0615 & 8.1012 & 0.4388 & 8.3951 \\
\hline & SS1_ML & 1.5156 & 6.4465 & 1.7864 & 1.8210 & 8.4117 & 0.4597 & 8.7274 \\
\hline & $\mathrm{SS} 2 \_\mathrm{ML}$ & 3.0231 & 58.2342 & 1.8345 & 1.9019 & 7.1528 & 27.9849 & 88.1210 \\
\hline & SSP_LS & 4.8160 & 12.7239 & 0.6559 & 0.0631 & 7.2409 & 1.6786 & 14.4656 \\
\hline & SS1_LS & 2.7116 & 9.3748 & 1.3902 & 1.4248 & 7.9054 & 1.2715 & 12.0711 \\
\hline & $\mathrm{SS} 2 \_\mathrm{LS}$ & 5.0092 & 200.6225 & 4.6330 & 81.3634 & 6.1252 & 53.3122 & 335.2982 \\
\hline \multirow[t]{6}{*}{30} & $\mathrm{SSP} \_\mathrm{ML}$ & 2.6957 & 0.5435 & 0.7177 & 0.0186 & 8.0675 & 0.0581 & 0.6203 \\
\hline & SS1_ML & 2.2893 & 3.2782 & 1.0215 & 0.2746 & 8.1479 & 0.1658 & 3.7187 \\
\hline & SS2_ML & 4.0812 & 89.1004 & 1.2096 & 0.4759 & 6.9153 & 28.4666 & 118.043 \\
\hline & SSP_LS & 3.0385 & 0.8608 & 0.7156 & 0.0200 & 7.8307 & 0.1638 & 1.0446 \\
\hline & $\mathrm{SS} 1 \_\mathrm{LS}$ & 2.9435 & 10.5749 & 1.1391 & 0.5477 & 7.7234 & 2.6769 & 13.7995 \\
\hline & $\mathrm{SS} 2 \_\mathrm{LS}$ & 7.0028 & 264.3197 & 3.5858 & 49.3927 & 3.8524 & 120.6242 & 434.3366 \\
\hline \multirow[t]{6}{*}{50} & $\mathrm{SSP} \_\mathrm{ML}$ & 2.4833 & 0.4581 & 0.7738 & 0.0156 & 8.0823 & 0.0467 & 0.5205 \\
\hline & SS1_ML & 2.3215 & 1.5162 & 0.9160 & 0.1202 & 8.1114 & 0.0821 & 1.7186 \\
\hline & SS2_ML & 4.2731 & 85.8568 & 1.0870 & 0.2848 & 6.6770 & 32.4816 & 118.6233 \\
\hline & SSP_LS & 2.9340 & 0.7428 & 0.7332 & 0.0135 & 7.8671 & 0.1209 & 0.8772 \\
\hline & $\mathrm{SS} 1 \_\mathrm{LS}$ & 3.0642 & 10.4554 & 1.0741 & 0.4427 & 7.6395 & 2.7570 & 13.6551 \\
\hline & $\mathrm{SS} 2 \_\mathrm{LS}$ & 8.5323 & 329.8226 & 3.5367 & 36.6483 & 1.6463 & 192.3202 & 558.7911 \\
\hline \multirow[t]{6}{*}{100} & $\mathrm{SSP} \_\mathrm{ML}$ & 2.4758 & 0.0937 & 0.7720 & 0.0061 & 8.0547 & 0.0131 & 0.1131 \\
\hline & SS1_ML & 2.3935 & 0.7622 & 0.8405 & 0.0435 & 8.0688 & 0.0372 & 0.8430 \\
\hline & SS2_ML & 4.0214 & 74.7848 & 1.0036 & 0.1822 & 6.9634 & 22.8519 & 97.8190 \\
\hline & SSP_LS & 2.5601 & 0.0737 & 0.7685 & 0.0065 & 8.0024 & 0.0193 & 0.0995 \\
\hline & SS1_LS & 3.2307 & 11.1256 & 0.9765 & 0.2974 & 7.6192 & 2.6028 & 14.0258 \\
\hline & $\mathrm{SS} 2 \_\mathrm{LS}$ & 8.1413 & 286.0229 & 3.6583 & 44.3889 & 1.8357 & 185.2244 & 515.6362 \\
\hline \multirow[t]{6}{*}{250} & SSP_ML & 2.4794 & 0.0677 & 0.7764 & 0.0034 & 8.0353 & 0.0064 & 0.0776 \\
\hline & $\mathrm{SS} 1 \_\mathrm{ML}$ & 2.4537 & 0.3922 & 0.8036 & 0.0164 & 8.0382 & 0.0214 & 0.4300 \\
\hline & $\mathrm{SS} 2 \_\mathrm{ML}$ & 4.0328 & 73.4884 & 0.9312 & 0.1048 & 6.8150 & 32.9088 & 106.5021 \\
\hline & SSP_LS & 2.5126 & 0.0596 & 0.7736 & 0.0032 & 8.0196 & 0.0095 & 0.0723 \\
\hline & $\mathrm{SS} 1 \_\mathrm{LS}$ & 3.3395 & 10.4881 & 0.9539 & 0.3893 & 7.5744 & 2.6145 & 13.4920 \\
\hline & $\mathrm{SS} 2 \_\mathrm{LS}$ & 9.5241 & 343.5391 & 4.1394 & 66.7236 & -2.1932 & 343.7817 & 754.0444 \\
\hline \multirow[t]{6}{*}{500} & $\mathrm{SSP} \_\mathrm{ML}$ & 2.5187 & 0.0351 & 0.7680 & 0.0017 & 8.0217 & 0.0029 & 0.0397 \\
\hline & SS1_ML & 2.4714 & 0.4105 & 0.7909 & 0.0095 & 8.0317 & 0.01942 & 0.4396 \\
\hline & $\mathrm{SS} 2 \_\mathrm{ML}$ & 4.3226 & 93.7741 & 0.9101 & 0.1215 & 6.9341 & 22.8579 & 116.7536 \\
\hline & SSP_LS & 2.5480 & 0.0346 & 0.7642 & 0.0016 & 8.0114 & 0.0059 & 0.0421 \\
\hline & $\mathrm{SS} 1 \_\mathrm{LS}$ & 3.3428 & 10.1838 & 0.9380 & 0.2983 & 7.5713 & 2.3045 & 12.7866 \\
\hline & $\mathrm{SS} 2 \_\mathrm{LS}$ & 9.9664 & 351.4923 & 3.6560 & 42.3463 & -2.5675 & 349.9287 & 743.7673 \\
\hline \multirow[t]{6}{*}{1000} & $\mathrm{SSP} \_\mathrm{ML}$ & 2.5509 & 0.0173 & 0.7601 & 0.0007 & 8.0142 & 0.0013 & 0.0195 \\
\hline & SS1_ML & 2.4914 & 0.1088 & 0.7795 & 0.0053 & 8.0262 & 0.0055 & 0.1198 \\
\hline & $\mathrm{SS} 2 \_\mathrm{ML}$ & 4.0531 & 70.6981 & 0.8856 & 0.0891 & 6.9009 & 25.7387 & 96.5267 \\
\hline & SSP_LS & 2.5654 & 0.0198 & 0.7583 & 0.0007 & 8.0092 & 0.0031 & 0.0237 \\
\hline & $\mathrm{SS} 1 \_\mathrm{LS}$ & 3.3702 & 9.4645 & 0.9322 & 0.3697 & 7.5570 & 2.2224 & 12.0566 \\
\hline & $\mathrm{SS} 2 \_\mathrm{LS}$ & 9.7969 & 351.3180 & 4.0083 & 57.9936 & -2.2125 & 337.2107 & 746.5223 \\
\hline
\end{tabular}

The Kruskal-Wallis test is used to examine the performance of the algorithms. While there is no significant difference in SSP search space at $95 \%$ confidence level for both methods ( $\mathrm{p}$ value for the $\mathrm{ML}=0.865$, $\mathrm{p}$-value for the $\mathrm{LS}=0.869$ ), significant differences are 
Table 10. Simulation results for the estimation of the parameters $\theta=(2.56,0.75,8)$ for $\mathrm{pop}=50$.

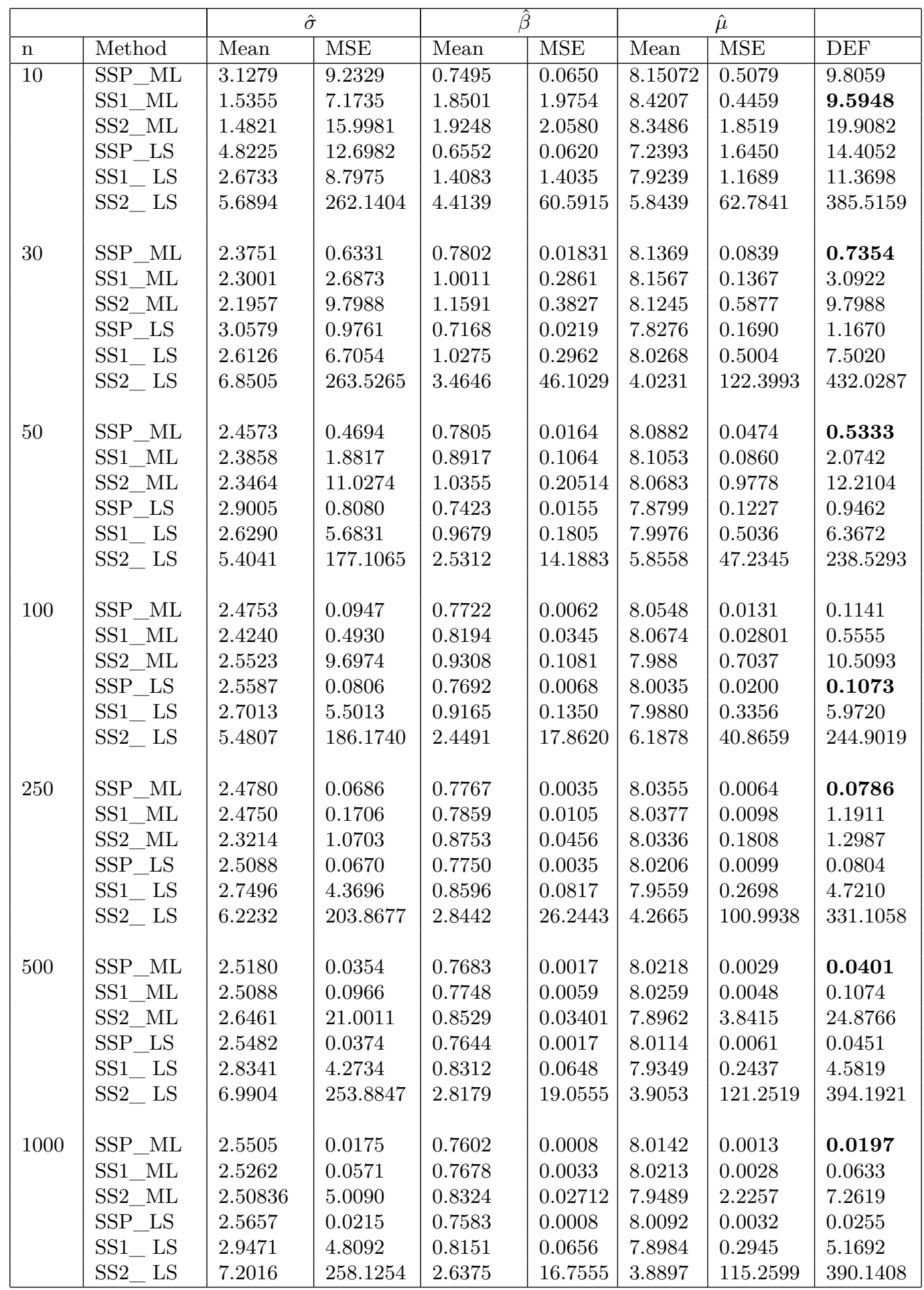

determined in SS1 and SS2 search spaces (p-value $=0.000$ for all cases). Furthermore, significant differences are found between $\mathrm{pop}=10$ and $\mathrm{pop}=25$, between $\mathrm{pop}=10$ and $\mathrm{pop}=50$ in SS1, and also between all population sizes in SS2.

Mann Whitney-U test is also conducted for testing whether there is a significant difference between the ML and LS estimates using the SSP search space. The p values for the 
sample sizes $\mathrm{n}=10,30,50,100,250,500$, and 1000 have been obtained as $0.004,0.136$, $0.258,0.863,0.666,0.340$, and 0.258 , respectively. It is found that, there is significantly difference between the ML and LS estimates only for $n=10$.

\section{Application}

In this section, the implementation of the proposed DE approach on the actual data set is investigated. The data set taken from experiments which are conducted in the Department of Materials Science and Engineering, the University of Surrey includes failure stresses of single carbon fibers and it is given below [8]:

$2.247 ; 2.640 ; 2.908 ; 3.099 ; 3.126 ; 3.245 ; 3.328 ; 3.355 ; 3.383 ; 3.572 ; 3.581 ; 3.681 ; 3.726$; $3.727 ; 3.72 ; 3.783 ; 3.785 ; 3.786 ; 3.896 ; 3.912 ; 3.964 ; 4.050 ; 4.063 ; 4.082 ; 4.111 ; 4.118 ; 4.141$; $4.246 ; 4.251 ; 4.26 ; 4.326 ; 4.402 ; 4.457 ; 4.466 ; 4.519 ; 4.542 ; 4.555 ; 4.614 ; 4.632 ; 4.634 ; 4.636$; $4.678 ; 4.698 ; 4.738 ; 4.83 ; 4.924 ; 5.043 ; 5.099 ; 5.134 ; 5.359 ; 5.473 ; 5.571 ; 5.684 ; 5.721 ; 5.998$; 6.060

To test the suitability of this data set for 3-p Gamma distribution, Kolmogorov Smirnov (K-S) goodness of fit test is used. For K-S test, null hypothesis and alternative hypothesis are given as follows:

$$
\begin{aligned}
& H_{o}: F(x)=F_{0}(x) \\
& H_{1}: F(x) \neq F_{0}(x)
\end{aligned}
$$

In this study, $F_{0}$ represents the distribution function for certain parameter values of 3-p Gamma distribution. The test statistic of KS is described by

$$
D=\sup \left(\left|F_{n}(x)-F_{0}(x)\right|\right)
$$

In Equation (4.2), $F_{n}(x)$ and $F_{0}(x)$ show the experimental and theoretical distribution functions at the sampling points of the Gamma distribution, respectively. At the confidence level $\alpha$, the hypothesis $H_{o}$ is rejected if the statistic $\mathrm{D}$ value is greater than or equal to the critical value $d_{\alpha}$, or if the calculated $\mathrm{p}$ value is less than $\alpha$. In this study, $\alpha=0.05$ is taken.

The values of $\log L$ and Akaike Information Criteria (AIC) have been used to show the performance of the proposed approach. The AIC is defined as follows:

$$
A I C=-2 \log L+2 k
$$

Here, $n$ is the number of recorded measurements, and $k$ is the number of estimated parameters.

$95 \%$ confidence intervals for the parameters of the 3-p Gamma distribution based on the MML and PL methods are obtained as $6.5998<\sigma<12.7820,0.2315<\beta<0.3391$ and $1.0188<\mu<1.9463$. These confidence intervals are used as the search space for each parameter. The ML and LS estimations of the parameters for the 3-p Gamma distribution are obtained by the DE algorithm with various search spaces. Parameter estimation values, $\log L, \mathrm{AIC}, \mathrm{K}-\mathrm{S}$ test statistics, and p-values for the considered methods are given in Table 11. 
Table 11. Parameter estimation values, $\log \mathrm{L}$, AIC, and K-S test statistics, and p-values for carbon fibre dataset.

\begin{tabular}{|l|l|l|l|l|l|l|l|}
\hline & $\hat{\sigma}$ & $\hat{\beta}$ & $\hat{\mu}$ & $\log \mathrm{A}$ & AIC & K-S & p-value \\
\hline SSP_ML & 12.7813 & 0.2481 & 1.0648 & -0.0441 & 6.0882 & 0.0750 & 0.9013 \\
SS1_ML & 8.2790 & 0.3475 & 1.5679 & -0.1765 & 6.3530 & 0.1344 & 0.2544 \\
SS2__ML & 5.9780 & 0.5236 & 1.7295 & -1.3667 & 8.7334 & 0.2731 & 0.0004 \\
SSP_LS & 12.3016 & 0.2421 & 1.3047 & $\mathbf{- 0 . 0 2 5 5}$ & $\mathbf{6 . 0 5 1 0}$ & 0.0620 & 0.9804 \\
SS1_LS & 9.4874 & 0.2850 & 1.6761 & -0.0900 & 6.1800 & 0.0980 & 0.6370 \\
SS2_LS & 8.1813 & 3.2249 & -1.5607 & -18.7883 & 43.5766 & 0.9976 & 0.0000 \\
\hline
\end{tabular}

According to the results of the K-S test, the distribution of the carbon fibre dataset fit the 3-p Gamma distribution since the p-values are greater than $\alpha$ except for the SS2_LML and the SS2 LS.

It is clear from Table 11, SSP has the largest $\log \mathrm{L}$ and the smallest AIC values for both the ML and LS. Thus, the SSP provides the most reliable results and the proposed approach should be preferred.

The histogram and the fitted density for carbon fibre data set for the ML and LS according to search space SSP and SS1, is shown in Figure 2 that supports the above results. In addition, it should be noted that SS1 can be used for the data set. However, it is more logical to use the SSP, since the arbitrarily determined SS1 may not include the actual parameter value for other datasets to be examined.

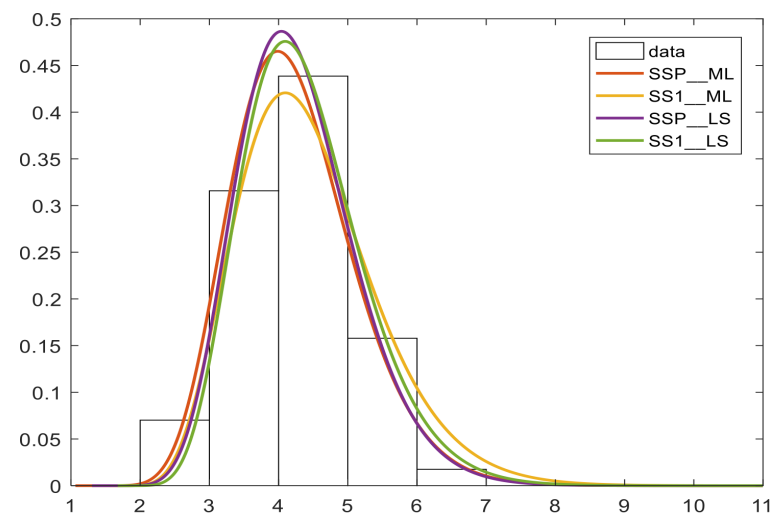

Figure 2. Histogram and fitted densities for the carbon fibre data set

\section{Conclusion}

This study discusses the ML and LS estimation problems of the parameters of the 3-p Gamma distribution. Because parameter estimation for the 3-p Gamma distribution is a quite difficult problem, it is wise to use a powerful metaheuristic method. Thus, the DE algorithm is used to find the ML and LS estimates of the parameters of the 3-p Gamma distribution. As with all metaheuristic methods, determination of the search space in $\mathrm{DE}$ is a very important problem. The performance of the DE is greatly affected by its search space in terms of both solution quality and computation time. In the literature, arbitrary search spaces have been traditionally used. However, the arbitrary search space may not contain the actual values and the global optimal solution cannot be found. To overcome this situation, a novel DE approach proposed in this study. In the proposed approach,confidence intervals based on the MML and PL methods are utilized for creating a search space for the DE algorithm which is narrow and has the actual values. 
To test the performance of this proposed new approach, an extensive Monte Carlo simulation study is performed. Simulation results show that the proposed DE algorithm approach gives significantly better results in both the ML and the LS estimation of the parameters. In addition, a real data set analysis is performed to demonstrate the applicability of the proposed DE approach. The superiority of the proposed approach is also supported by real data set.

As a future research, the proposed DE approach can be applied to estimate the parameters of different statistical distributions. Furthermore, different metaheuristic methods can be used for estimating the parameters of the 3-p Gamma distribution in order to compare the solutions of the proposed DE approach.

Acknowledgment. This study is a part of PhD thesis of Aynur YONAR titled "Metaheuristic Approaches for Estimating Parameters of Univariate and Multivariate Distributions", which is in progress at the Selcuk University, Graduate School of Natural and Applied Sciences, Department of Statistics, Konya, Turkey. This PhD thesis has been supported by Faculty Development Program (FDP) with Project No: 2016-OYP-063.

\section{References}

[1] Ş. Acıtaş, Ç.H. Aladă̆, and B. Şenoglu, A new approach for estimating the parameters of Weibull distribution via particle swarm optimization: An application to the strengths of glass fibre data, Reliability Engineering System Safety, 183, 116-127, 2019 .

[2] M. Asim, W.M. Khan, Ö. Yeniay, M. A. Jan, N. Tairan, H. Hussian, and G.-G. Wang, Hybrid genetic algorithms for global optimization problems. Hacet. J. Math. Stat., 47 (3), 539-551, 2018.

[3] N. Balakrishnan and J., Wang, Simple efficient estimation for the three-parameter gamma distribution, J. Statist. Plann. Inference, 85 (1-2), 115-126, 2000.

[4] I. Başak and N. Balakrishnan, Estimation for the three-parameter gamma distribution based on progressively censored data, Stat. Methodol., 9 (3), 305-319, 2012.

[5] O.T. Bayrak and A.D. Akkaya, Autoregressive models with stochastic design variables and nonnormal innovations, Recent Researches in Applied Mathematics, Simulation and Modeling, Proceedings of the 5th International Conference on Applied Mathematics, Simulation, Modeling, 197-201, 2011.

[6] A.C. Cohen and B.J. Whitten, Modified moment and maximum likelihood estimators for parameters of the three-parameter gamma distribution, Comm. Statist. Simulation Comput., 11 (2), 197-216, 1982.

[7] A.C. Cohen and B.J. Whitten, Modified moment estimation for the three-parameter gamma distribution, Journal of Quality Technology, 18 (1), 53-62, 1986.

[8] M.J. Crowder, A.C. Kimber, R.L. Smith, and T.J. Sweating, The Statistical Analysis of Reliability Data, Chapman and Hall, London, 1991.

[9] S. Das, S.S. Mullick, and P.N. Suganthan, Recent advances in differential evolutionan updated surve, Swarm Evolutionary Computation, 27, 1-30, 2016.

[10] H. Hirose, Maximum likelihood parameter estimation in the three-parameter gamma distribution, Comput. Statist. Data Anal., 20,(4) 343-354, 1995.

[11] N.L. Johnson, S. Kotz, and N. Balakrishnan, Univariate continuous distributions: New York: John Wiley \& Sons, 1994.

[12] V. Lakshmi and V. Vaidyanathan, Three-parameter gamma distribution: Estimation using likelihood, spacings and least squares approach, Journal of Statistics Management Systems, 19 (10), 37-53, 2016.

[13] W.K. Mashwani, Enhanced versions of differential evolution: state-of-the-art survey, Int. J. Comput. Sci. Math., 5 (2), 107-126, 2014. 
[14] W.K. Mashwani, et al., Hybrid Constrained Evolutionary Algorithm for Numerical Optimization Problems. Int. J. Comput. Sci. Math., 48 (3), 931-950, 2018.

[15] E. Mezura-Montes, M.E. Miranda-Varela, and R. Carmen Gomez-Ramon, Differential evolution in constrained numerical optimization: an empirical study. Inform. Sci., 180 (22), , 4223-4262, 2010.

[16] A.W. Mohamed and H.Z. Sabry, Constrained optimization based on modified differential evolution algorithm, Inform. Sci., 194, 171-208, 2012.

[17] M.N. Omidvar, X. Li, Y. Mei, and X. Yao, Cooperative co-evolution with differential grouping for large scale optimization. IEEE Trans. Evol. Comput., 18(3), 378-393, 2013.

[18] E.O.J. Ouedraogo, B. Some, and S. Dossou-Gbete, On Maximum Likelihood Estimation for the Three Parameter Gamma Distribution Based on Left Censored Samples, Sci. J. Appl. Math. and Stat., 5(4), 147-163, 2017.

[19] H. Örkçü, E. Aksoy, and M.I. Doğan, Estimating the parameters of 3-p Weibull distribution through differential evolution, Appl. Math. Comput., 251, 211-224, 2015.

[20] V.S. Özsoy, M.G. Ünsal, and H.H. Örkçü, Use of the heuristic optimization in the parameter estimation of generalized gamma distribution: comparison of GA, DE, PSO and SA methods, Comput. Statist. Data Anal., 1-31, 2020.

[21] K. Price, R.M. Storn, and J.A. Lampinen, Differential evolution: a practical approach to global optimization: Springer Science and Business Media, 2006.

[22] K. Sindhya, S. Ruuska, T. Haanpaa, and K. Miettinen, A new hybrid mutation operator for multiobjective optimization with differential evolution, Soft Computing, 15 (10), 2041-2055, 2011.

[23] R. Storn, On the usage of differential evolution for function optimization. in: Fuzzy Information Processing Society, Biennial Conference of the North American, 519-523, IEEE, 1996.

[24] R. Storn and K. Price, Differential evolutiona simple and efficient heuristic for global optimization over continuous spaces, J. Global Optim., 11 (4), 341-359, 1997.

[25] E.-G. Talbi, Metaheuristics: from design to implementation: John Wiley \& Sons, 2009.

[26] M.L. Tiku and A.D. Akkaya, Robust estimation and hypothesis testing: New Age International, 2004.

[27] G. Tzavelas,Maximum likelihood parameter estimation in the three-parameter gamma distribution with the use of Mathematica. J. Stat. Comput. Simul., 79 (12), 1457-1466, 2009.

[28] G. Tzavelas, Estimation in the Three-Parameter Gamma Distribution Based on the Profile Log-Likelihood Function, Comm. Statist. Theory Methods, 38 (5), 573-583, 2009.

[29] D. Vaughan and M. Tiku, Estimation and hypothesis testing for a nonnormal bivariate distribution with applications. Math. Comput. Model., 32 (4), 27, 2011. (1-2), 53-67, 2000.

[30] A. Yalçınkaya, B. Şenoglu, and U. Yolcu, Maximum likelihood estimation for the parameters of skew normal distribution using genetic algorithm, Swarm and Evolutionary Computation, 38, 127-138, 2018.

[31] X.-S. Yang, Engineering optimization: An introduction with metaheuristic applications: John Wiley \& Sons, 2010.

[32] J.-H. Zhong and J. Zhang, SDE: A stochastic coding differential evolution for global optimization in:Proceedings of the 14th annual conference on Genetic and evolutionary computation, 975-982, ACM, 2012. 